\title{
A lower bound on the Milky Way mass from general phase-space distribution function models ${ }^{\star}$
}

\author{
Łukasz Bratek $^{1}$, Szymon Sikora ${ }^{2}$, Joanna Jałocha ${ }^{1}$, and Marek Kutschera ${ }^{3}$ \\ ${ }^{1}$ Institute of Nuclear Physics, Polish Academy of Sciences, Radzikowskego 152, 31342 Kraków, Poland \\ e-mail: Lukasz.Bratek@ifj.edu.pl \\ 2 Astronomical Observatory, Jagiellonian University, Orla 171, 30244 Kraków, Poland \\ 3 Institute of Physics, Jagiellonian University, Reymonta 4, 30059 Kraków, Poland
}

Received 5 September 2013 / Accepted 4 December 2013

\begin{abstract}
We model the phase-space distribution of the kinematic tracers using general, smooth distribution functions to derive a conservative lower bound on the total mass within $\approx 150-200 \mathrm{kpc}$. By approximating the potential as Keplerian, the phase-space distribution can be simplified to that of a smooth distribution of energies and eccentricities. Our approach naturally allows for calculating moments of the distribution function, such as the radial profile of the orbital anisotropy.

We systematically construct a family of phase-space functions with the resulting radial velocity dispersion overlapping with the one obtained using data on radial motions of distant kinematic tracers, while making no assumptions about the density of the tracers and the velocity anisotropy parameter $\beta$ regarded as a function of the radial variable.

While there is no apparent upper bound for the Milky Way mass, at least as long as only the radial motions are concerned, we find a sharp lower bound for the mass that is small. In particular, a mass value of $2.4 \times 10^{11} M_{\odot}$, obtained in the past for lower and intermediate radii, is still consistent with the dispersion profile at larger radii. Compared with much greater mass values in the literature, this result shows that determining the Milky Way mass is strongly model-dependent. We expect a similar reduction of mass estimates in models assuming more realistic mass profiles.
\end{abstract}

Key words. techniques: radial velocities - Galaxy: halo - Galaxy: kinematics and dynamics - Galaxy: fundamental parameters methods: numerical

\section{Introduction}

The asymptotic value of galactic mass function (total mass) can be ascertained through studying the radial motions of distant kinematic tracers, which are regarded as test bodies moving under the influence of the galactic gravitational field. A primary quantity for describing a collection of these bodies is a phasespace distribution function (PDF) which must be non-negative everywhere. The function gives rise to various theoretical secondary quantities such as the radial velocity dispersion (RVD), the flattening of the velocity ellipsoid $\beta$ (the anisotropy parameter), the number density of tracers, mean velocities, etc, which in general may be functions of space variables defined as appropriate integrals involving the PDF. On comparison with the corresponding quantities from measurements, the total mass can be inferred.

The accustomed approach to this technique of determining the total mass is based on Jeans modeling of a stationary collision-less system of identical bodies in a steady-state equilibrium $^{1}$ (Jeans 1915). Jeans concludes his work by saying that the Galaxy has not yet reached a final steady state on account of the fact that such a state seems to be inconsistent with the observed streaming motions. The principal assumption of this technique requires that the kinematic tracers are relaxed in the gravitational potential and can be described in terms of a smooth

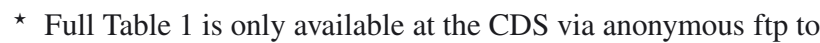
cdsarc.u-strasbg.fr (130.79.128.5) or via http://cdsarc.u-strasbg.fr/viz-bin/qcat?]/A+A/562/A134 $1 \partial_{t} f \equiv 0$ (steady-state); $d_{t} f \equiv 0$ (equilibrium) for a $\operatorname{PDF} f$.
PDF. However, the assumption of collision-less equilibrium may not necessarily be appropriate. In particular, this may be the case both for fast moving tracers (e.g., propelled by the three-body ejecting mechanism, which is collisional in its nature) and for most distant tracers, on orbits characterized by large time scales, which are likely to be influenced by the material distributed on larger scales in the Local Group. It is also doubtful that the very notion of a smooth distribution is applicable to an extremely rarefied collection of the outermost satellites of the Milky Way (MW). Although Jeans' modeling is a powerful tool that was fruitful in many cases, it should be remembered that it has a limited application.

In recent years, there has been growing interest in the most general distributions functions that can be fit to dynamical data with most general and assumption-free constraints on the gravitational potential and mass profile. Under the assumption that a galaxy is in a steady state, Magorrian (2014) proposes a new framework of inferring the gravitational potential from a discrete realization of the unknown distribution function provided by the snapshots of a galaxy's stellar kinematics. Bovy et al. (2010) used instantaneous kinematic snapshots of radial distances and velocities to infer the force law with a fully Bayesian inference technique. As an example, when applying it to the solar system, the correct exponent in the power form of the force law was almost precisely reconstructed, largely independent of the PDF of two variables (energy and eccentricity) with no need to make strong assumptions about the anisotropy. As noted in Bovy et al. (2010), generalizations of the methods used therein would permit inference of MW dynamics from upcoming surveys, such as 
the Gaia mission (Perryman et al. 2001), or of the mass of the central black hole.

In this paper, the form of PDF is also general. Its form is not assumed except that it should be a function of integrals of motion to automatically satisfy the Boltzmann equation. Our aim is to find, in a given gravitational potential that allows for such integrals, a variety of PDFs consistent with the radial distancevelocity data. In this preliminary work, we do not make any assumptions about the form of other secondary observables derivable from a given PDF (such as the transverse velocity dispersion or the profile of the anisotropy parameter) that could be used to constrain the variety of consistent PDFs. We focus instead on the resulting estimate of the MW's minimum total mass.

The secondary quantities referred to above are not all independent. They are constrained to satisfy (moment) Jeans equations. However, satisfying them is not sufficient for the positivity of the PDF. In principle, having found a solution, the positivity condition should be checked separately. Jeans equations are usually underdetermined - there is arbitrariness in choosing solutions. Some of the secondary quantities must be assumed (along with the mass function of the host gravitational field). This makes the problem of determining the total mass model-dependent and biased by inescapable degeneracies. The existing mass estimates can differ largely between each other. The differences lie both on the side of choosing sample tracers and making model assumptions. In the context of this underdeterminacy, one cannot exclude that masses predicted by various models can be overestimated. The mechanism of this overestimation can be understood as follows. The total mass can be regarded as a functional defined on the space of solutions to the Jeans equation ${ }^{2}$. As said above, various quantities entering that equation must involve additional assumptions. These assumptions are introduced in the form of constraints, such as a constant value for $\beta$, a power law for the number density, and the particular form of the gravitating mass function of the host potential. These constraints impose some indirect restrictions on the PDF function. As for any functional with constraints, so also for the total mass functional, one can expect that the lower bound may be increased compared to that without constraints ${ }^{3}$.

From the above consideration, a natural question arises for the lower bound of the MW mass. This question motivates our paper. Our aim is to show that with a given set of radial motions of kinematic tracers, the estimated mass can be much lower than usually obtained in the literature. We postpone answering this question until Sects. 3 and 4.

Another, but related, question concerns the upper bound. It may be answered more straightforwardly. We consider the point mass potential - the asymptotics of any physical mass distribution. The time average $\left\langle r v_{r}^{2}\right\rangle_{t}=\frac{1}{2} G M e^{2}$ for a single elliptical orbit with eccentricity $e$, suggests that $\left\langle v_{r}^{2}\right\rangle \sim r^{-1}$ for almost circular orbits. Assuming this behavior of $\left\langle v_{r}^{2}\right\rangle$ in the spherical Jeans equation $\frac{r \rho_{r}}{\rho}+2 \beta=-\frac{r}{\left\langle v_{r}^{2}\right\rangle}\left(\Phi+\left\langle v_{r}^{2}\right\rangle\right)_{, r}$, along with the vanishing

\footnotetext{
2 For example, the gravitational energy of a virialized self-gravitating dust is equal to minus twice the total kinetic energy. On dimensional grounds, the binding energy is a product of the total mass squared and the inverse of some characteristic size determined by the spatial distribution of dust. The total mass of a virialized system is thus a functional on the phase-space.

3 A good example of determining lower bounds with constraints is provided by the minimum-maximum method of finding consecutive eigenvalues and eigenvectors of a quadratic form in a finite number of dimensions or, in an infinitely dimensional function space, of a self-adjoint differential equation (Courant \& Hilbert 1953).
}

mean velocities, constant $\beta=1-\frac{\left\langle v_{\theta}^{2}\right\rangle+\left\langle v_{\phi}^{2}\right\rangle}{2\left\langle v_{r}^{2}\right\rangle}$, and $\rho \sim \rho_{\mathrm{o}}\left(r_{\mathrm{o}} / r\right)^{3+\varepsilon}$ $(\varepsilon>0)$, we conclude that asymptotically, when $r \Phi \sim-G M$, the mass estimate for almost circular orbits is

$M \sim \mu G^{-1} r\left\langle v_{r}^{2}\right\rangle \quad$ with $\quad \mu=4+\varepsilon-2 \beta>4-2 \beta>2$.

This result holds without the need for the phenomenological explicit assumption that $\left\langle v_{r}^{2}\right\rangle \sim r^{-1}$. A general integral of the spherical Jeans equation found in An \& Evans (2009) under similar assumptions about $\beta$ and $\rho$, reduces in the point mass potential to $\left\langle v_{r}^{2}\right\rangle=\frac{G M}{4-2 \beta+\varepsilon} r^{-1}+C r^{4-2 \beta+\varepsilon}$ (in our notational convention), where $C$ is an integration constant. In the asymptotical regime we work in, the appropriate boundary condition is to require that $\left\langle v_{r}^{2}\right\rangle$ should be finite in the limit $r \rightarrow \infty$, which for $4-2 \beta+\varepsilon>0$ implies that $C=0$, again giving (1). Result (1) says that an almost flat $r\left\langle v_{r}^{2}\right\rangle$ profile could be explained by arbitrary large $M$ ( $\beta$ and $\varepsilon$ are not controlled by measurements at large radii ${ }^{4}$ ). There is no apparent upper bound for the mass. This will become even more evident in Sect. 4.

In the asymptotic consideration above, the presence of arbitrary parameters (such as $\varepsilon$ and $\beta$ ) represents the freedom in choosing solutions in more general situations. As we have seen, the relation between the mass function and the RVD profile must be arbitrary to some extent, because the kinematics is not entirely linked to the mass distribution. This is the important degeneracy of the Jeans problem: the inferred mass depends on the assumed model parameters (especially $\beta$ in the above example). In other words, the asymptotics of solutions is not entirely fixed by measurements in the interior, so some analytic continuation of solutions must be assumed. This indeterminacy is physically clear. First, the motions are not entirely due to the massive (monopole) term in the potential. Higher multipoles also affect the motion, despite being massless components of the gravitational field. Second, in a given potential one can consider infinitely many ensembles of test bodies with various stationary velocity dispersion profiles (evolved from various sets of initial data). There are some conserved quantities (e.g., total energy) that constrain the evolution in the phase-space, therefore, the (collisionless) relaxation to a stationary state cannot lead to a universal dispersion profile. The profile should be a functional of the initial data.

With general solutions to the Jeans problem, the estimated mass value can be reduced effectively. We show this in Sect. 3 in the approximation of a point mass field. The idea of studying the motions of external tracers in a point-mass approximation should not be surprising. It was already considered over 30 years ago by Bahcall \& Tremaine (1981) and applied to several external galaxies. The authors proposed an estimator for the galactic mass of the form $\frac{C}{G}\left\langle v_{z}^{2} R\right\rangle$, where the average is taken over compact halo objects (in cylindrical coordinates), and $C$ is a constant parameter. The parameter depended on the assumptions about the form of the PDF and on a mean square of the eccentricity. Also, Little \& Tremaine (1987) modeled the MW as a point. A recent paper (Watkins et al. 2010) offers essentially the same method as in Bahcall \& Tremaine (1981), and the difference lies mainly in the arbitrary power of the radial distance in the mass estimator. Using estimators of this kind is tantamount to considering a very particular family of PDFs. Interestingly, by playing with various assumptions, the authors found that the MW mass could be as low as $4 \times 10^{11} M_{\odot}$ (but also as high as $\left.2.7 \times 10^{12} M_{\odot}\right)$. Wilkinson \& Evans (1999) used a model

4 It is not inconceivable that we can make in the nearest future more precise measurements of parameters $\beta$ and $\varepsilon$ at the outermost radii, in which case solutions of the Jeans equation would be more robust. 
for the MW halo with variable $\beta$. Having applied it to a sample of satellites with known proper motions, they found the mass within $50 \mathrm{kpc}$ to be $5.4_{-3.6}^{+0.2} \times 10^{11} M_{\odot}$ (unaffected by the presence or absence of Leo I). The limits set on the mass within $50 \mathrm{kpc}$ in Sakamoto et al. (2003) with a larger number of tracers are similarly unaffected by Leo I: $5.5_{-0.2}^{+0.0} \times 10^{11} M_{\odot}$ (with Leo I) and $5.4_{-0.4}^{+0.1} \times 10^{11} M_{\odot}$ (without Leo I). Mass models considered in Klypin et al. (2002) give 5.8-6.0 $\times 10^{11} M_{\odot}$ within $100 \mathrm{kpc}$. A more recent estimate are $4.2 \times 10^{11} M_{\odot}$ within $50 \mathrm{kpc}$ (Deason et al. $2012 \mathrm{a}$ ) or $3.5-5 \times 10^{11} M_{\odot}$ within $150 \mathrm{kpc}$ for a Keplerian ${ }^{5}$ halo model (Deason et al. 2012b) based on the same estimator as in Watkins et al. (2010). These results substantiate a low-mass possibility within $150 \mathrm{kpc}$.

It is interesting to ask if the mass could be reduced further and still account for the RVD profile. In Sect. 3 under spherical symmetry, we allow for a general PDF, which is a function of two integrals of motion (the energy and the eccentricity) that describe a continuous collection of confocal elliptical orbits. To make the approximation legitimate, we cut the support of the PDF off, so that the orbits' perycentra are bounded from below by the radius of a spherical shell encompassing the Galactic disk. Then the secondary quantities, such as the theoretical RVD, are obtained directly from the PDF and can be quite general functions of the radial variable. A particular PDF is found by minimizing the discrepancy between the theoretical and the measured RVD profiles. In this paper we do not impose constraints on other secondary quantities, such as the density and anisotropy profiles of the tracers. The task of determining the PDF requires a good deal of numerical work. A procedure for obtaining the PDF is discussed briefly in Sect. 3.1.3. In Sect. 3 a detailed description of the theory behind this procedure is given.

As an example of using our method in practice, we apply it to estimate the lower bound for the MW mass. To this end we use a sample of tracers that are likely to be bound to the MW if its mass is not greater than $3.5 \times 10^{11} M_{\odot}$, a value that coincides with the lowest mass estimate in the point-mass field within $150 \mathrm{kpc}$, as recently obtained for a Keplerian halo model (Deason et al. 2012b). This value is also consistent with another mass estimate (without Leo I) obtained by Wilkinson \& Evans (1999) for a model with variable anisotropy parameter and integrable halo mass. Dark halo profiles in the literature are mostly nonintegrable. Our aim is to show that the mass can be reduced further.

Finally, we present an example result for the same sample, assuming the central mass of $2.4 \times 10^{11} M_{\odot}$. This value is chosen for two reasons: it is greater than the lower bound we find, and it was already obtained in the past: a) in a three-component mass model with an asymptotic rotation velocity $230 \mathrm{~km} \mathrm{~s}^{-1}$ of the dark halo, fitted to the rotation of the HI layer extending to $\approx 20 \mathrm{kpc}$ (Merrifield 1992); and b) as the best estimate at the $68 \%$ confidence level obtained assuming an isotropic velocity distribution in the point mass field for a sample of satellites at distances of $50-140 \mathrm{kpc}$ Little \& Tremaine (1987, where a reservation was made that the estimate could be lower with more radial orbits).

\section{Position-velocity data}

We assume the following parameters: $R_{\circ}=8.5 \pm 0.4 \mathrm{kpc}$ for the Sun's distance from the Galactic center, $V_{\circ}=240 \pm$ $16 \mathrm{~km} \mathrm{~s}^{-1}$ for the local disk rotation speed based on three

\footnotetext{
5 Here, "Keplerian" means $M=\frac{C}{G}<r^{\gamma} v_{r}^{2}>$ with $\gamma=1$.
}

estimates $\left(244 \pm 13 \mathrm{~km} \mathrm{~s}^{-1}\right.$ from maser data and the motion of $\operatorname{SgrA}^{*}$ (Bovy et al. 2009), $V_{\circ}=221 \pm 18 \mathrm{~km} \mathrm{~s}^{-1}$ from GD-1 stellar stream (Koposov et al. 2010), and $254 \pm$ $16 \mathrm{~km} \mathrm{~s}^{-1}$ another estimate from masers). We assume $(U, V, W)=$ $(11.1 \pm 1.7,12.24 \pm 2.5,7.25 \pm 0.9) \mathrm{km} \mathrm{s}^{-1}$ for the components of the velocity vector of the Sun with respect to the local standard of rest based on Schönrich et al. (2010) who give $U=$ $11.1_{-0.75}^{+0.69}, V=12.24_{-0.47}^{+0.47}, W=7.25_{-0.36}^{+0.37}$ with the additional systematic uncertainties $(1,2,0.5)$. For a summary of other measurements, see Francis \& Anderson (2009).

To prepare the radial velocity dispersion profile (RVD), which is a sequence of averages $\left\langle r v_{r}^{2}\right\rangle$ over concentric spherical shells of increasing size, we used the following positionvelocity data: the catalogs of halo giant stars (Dohm-Palmer et al. 2001; Starkenburg et al. 2009) based on the Spaghetti Project Survey (Morrison et al. 2000); the database of blue horizontal branch stars (Clewley et al. 2004) from United Kingdom Schmidt Telescope observations and SDSS; the database of field horizontal branch and A-type stars (Wilhelm et al. 1999) based on the survey of Beers et al. (1992); the catalogs of globular clusters (Harris 1996) and dwarf galaxies (Mateo 1998). The data was recalculated to epoch J2000 when necessary. In addition, we included the ultra-faint dwarf galaxies such as Ursa Major I and II, Coma Berenices, Canes Venatici I and II, Hercules (Simon \& Geha 2007), Bootes I, Willman 1 (Martin et al. 2007), Bootes II (Koch et al. 2009), Leo V (Belokurov et al. 2008), Segue I (Geha et al. 2009), and Segue II (Belokurov et al. 2009). To eliminate a possible decrease in the RVD at smaller radii due to circular orbits in the disk, we excluded tracers in the ellipsoidal disk vicinity: $\frac{Z^{2}}{a^{2}}+\frac{R^{2}}{b^{2}} \leq 1$ with $a=4 \mathrm{kpc}$ and $b=20 \mathrm{kpc}$. Here, $20 \mathrm{kpc}$ agrees with the extension of the observable disk (Carney 1984), while $4 \mathrm{kpc}$ at smaller radii agrees with a criterion used by Xue et al. (2008) to exclude some thick-disk stars for which $|Z|<4 \mathrm{kpc}$. We excluded Leo T located at $r>400 \mathrm{kpc}$ (its large spatial separation from closer tracers makes it unsuitable for preparing the RVD profile).

There is a large uncertainty in the MW mass determination, especially at large distances $\gtrsim 50 \mathrm{kpc}$, mainly due to the poorly constrained spatial extent of the dark halo, dominating baryonic mass components. Total mass estimates can differ by a factor of 4 or more, ranging from $0.5 \times 10^{12} M_{\odot}$ to $2 \times 10^{12} M_{\odot}$ (Brown et al. 2010) and more. The dark halo's status is entirely speculative (Dehnen \& Binney 1998). As a result, it is not a priori known which of high-velocity tracers are gravitationally bound to the MW. Mass estimates of the MW are largely affected by several such tracers (Sakamoto et al. 2003). Apart from Leo I these are: Pal 3, Draco, and a few FHB stars. However, Leo I seems almost certainly unbound, as the argument below shows.

\subsection{The case of Leol}

Leo I is a dwarf spheroidal galaxy, a very distant and fastreceding satellite of the MW. As follows from the escape velocity argument, Leo I could be bound to the MW, if MW's mass was greater than $\frac{1}{2 G} r\left(v_{\text {rad }}^{2}+v_{\tan }^{2}\right)=1.16 \times 10^{12} M_{\odot}$, where we have used values given in Sohn et al. (2013). As can be seen in Fig. 1, the central mass should be much higher than $2 \times 10^{12} M_{\odot}$, if one agrees that the orbital period of a relaxed bound object should be much lower than the Hubble time. (Leo I is often included in the Jeans analysis which by definition assumes a stationary relaxed system of tracers.) Furthermore, it would be unreasonable to expect the apocenter of a MW's satellite (that is, a body not affected gravitationally by the presence 

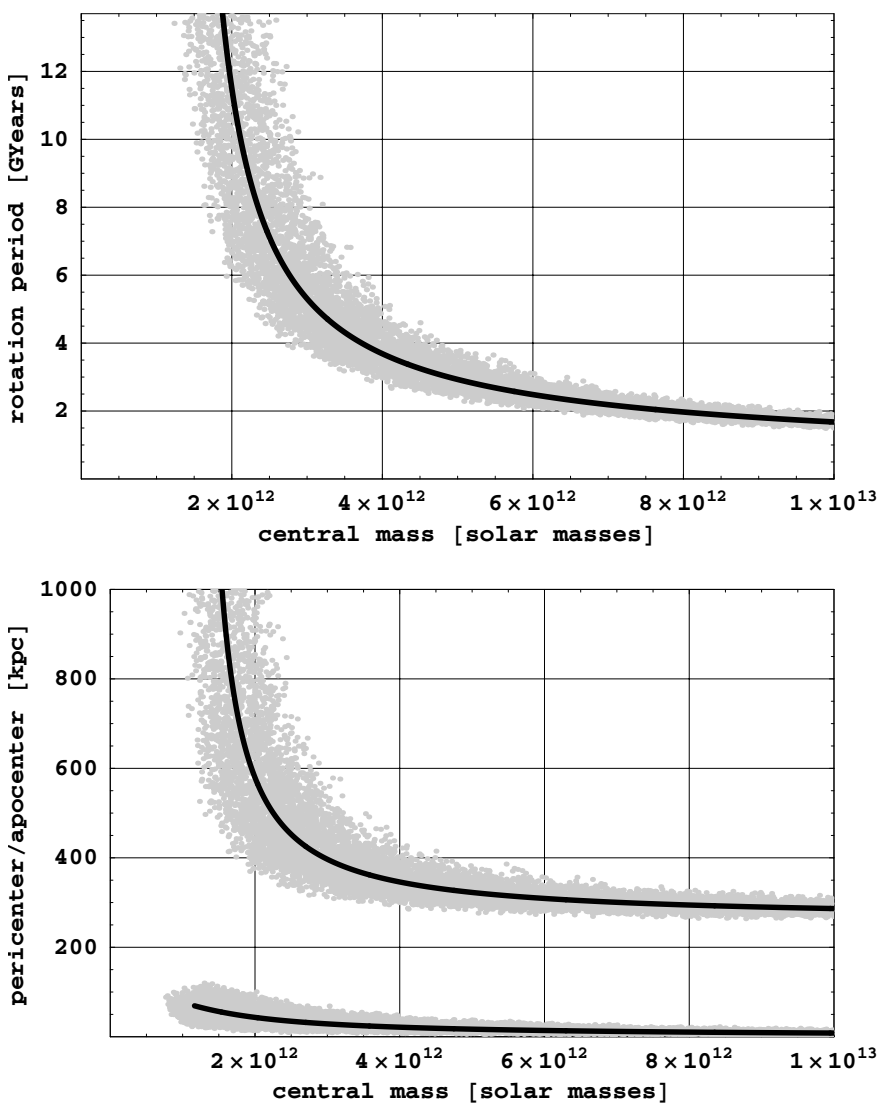

Fig. 1. Orbital period, pericenter, and apocenter for Leo I shown as functions of the central mass in the point mass potential, calculated according to Leo I's Galactocentric position and velocity given in Sohn et al. (2013). The gray dots represent random values for bound (elliptic) orbits admissible within errors.

of other neighboring high-mass concentrations, like M31) to be close to, or further than halfway distance between large galaxies in the Local Group, which is $\approx 350 \mathrm{kpc}$. This gives at least $3 \times 10^{12} M_{\odot}$ for the MW mass, as seen from Fig. 1 . Also, the timing argument (Kahn \& Woltjer 1959) leads to a comparable mass $M_{\text {vir }}=3.15_{-1.36}^{+1.58} \times 10^{12} M_{\odot}$ (Sohn et al. 2013). But a timing argument is likely to overestimate the true mass, as van der Marel \& Guhathakurta (2008) noticed for the M31-MW system, even by a factor of 2 . Such high mass is also improbable according to the argument by Wang et al. (2012): the probability that MW's $\Lambda \mathrm{CDM}$ halo has the observed number of subhalos with a given maximum circular velocity, decreases steeply with increasing MW's virial mass, effectively vanishes for $M_{\mathrm{vir}}>3 \times 10^{12} M_{\odot}$, and is only $5 \%$ for $M_{\text {vir }}>2 \times 10^{12} M_{\odot}$. Therefore, the MW mass cannot be too high, so the timescale for Leo I must be within an order of magnitude of the Hubble time. This contradicts the assumption that the time scale is much lower.

High masses from the timing argument are also inconsistent with recent mass estimates. The favored model in (Klypin et al. 2002) gives $10^{12} M_{\odot}$ for the virial mass of the MW. Xue et al. (2008) estimate the MW mass to $4.0 \pm 0.7 \times 10^{11} M_{\odot}$ within $60 \mathrm{kpc}$ from the kinematics of a large virialized sample of BHB stars, and extrapolate this result to the MW's dark halo mass $M_{\mathrm{vir}}=10_{-2}^{+3} \times 10^{11}$. Deason et al. (2012b) suggest that the mass within $150 \mathrm{kpc}$ is probably in the range from $5.0 \times 10^{11} M_{\odot}$ to $10.0 \times 10^{11} M_{\odot}$ and that there may be low mass between 50 and $150 \mathrm{kpc}$ (implying a high-concentration halo). The authors come to the conclusion that Leo I is almost certainly unbound. Next, Di Cintio et al. (2013) conclude with the MW's mass is between 5.5-7.5 $\times 10^{11} M_{\odot}$. Another recent work (Vera-Ciro et al. 2013) finds that the number and internal dynamics of the classical dwarf spheroidal satellites will be consistent with the predictions of the $\Lambda \mathrm{CDM}$ model, if the MW total mass is $8.0 \times 10^{11} M_{\odot}$, again insufficient to bind Leo I to the MW. The latter value was obtained in the past as the upper bound for the mass at the $99 \%$ confidence level, by assuming an isotropic velocity distribution for objects at distances of 50-140 kpc (Little \& Tremaine 1987), with the reservation that this estimate can be lower for more radial orbits.

Leo I is a kinematical outlier from the rest of velocity tracers. In this context we recall that the statistical analysis of data advises not including single measurements that evidently are well outside the range of other data. It would hardly be an acceptable situation if the MW mass was determined using a single tracer (while it is uncertain whether it is bound or not). By the mere assumption that Leo I is bound, one would have to accept a lower limit for the MW mass of at least $1.16 \times 10^{12}$, even though motions of a vast majority of remaining tracers or other arguments as in Wang et al. (2012) might point to a lower mass, insufficient to bind Leo I. As tested by Deason et al. (2011), including unbound satellites in two popular mass estimates (Bahcall \& Tremaine 1981; Watkins et al. 2010) can cause large overestimates of the true mass. Leo I disproportionately affects MW mass estimates under the assumption of equilibrium kinematics (Sohn et al. 2013), e.g. by adding Leo I, the mass estimate can be increased by nearly a factor of 3 (Zaritsky et al. 1989). A simple mass estimator applied to the satellite populations (Leo I discarded) gives the minimum $4 \pm 1 \times 10^{11} M_{\odot}$ for enclosed mass within $300 \mathrm{kpc}$ (Watkins et al. 2010), whereas including Leo I would increase the estimate to $15.0 \pm 4.0 \times 10^{11} M_{\odot}$. By using a halo model (Wilkinson \& Evans 1999) with variable anisotropy parameter and integrable halo mass, one can show that both the mass and the length scale change a lot: when Leo I is included the most likely values are $17 \times 10^{11} M_{\odot}$ and $150 \mathrm{kpc}$ for the total halo mass and the length scale, whereas with Leo I excluded, the quantities shrink by a factor of 6 to $3.0 \times 10^{11} M_{\odot}$ and $25 \mathrm{kpc}$. According to us, determination of mass should be stable against inclusion/exclusion of a sufficiently small subsamples of velocity tracers. With Leo I this is by no means possible.

As it was suggested in Sohn et al. (2013), there might be a $77 \%$ chance that Leo I could be bound to the MW. However, the same authors conclude that it would not necessarily be appropriate to include Leo I in equilibrium models used to estimate the MW virial mass. The kinematics of Leo I is unlikely to be virialized on account of its expected first infall into the MW. It was concluded in Byrd et al. (1994), that the history of the local group is too complex to justify calculating the mass of the MW by assuming that all satellites of our galaxy are bound. Second, a more prosaic scenario cannot be excluded: Leo I may have passed coincidentally by us, and could have originated outside the MW (Zaritsky et al. 1989). Third, as observed in Sales et al. (2007), fast receding satellites, such as Leo I, can be present owing to a three-body ejection mechanism, which is propelling bodies into highly energetic orbits.

Given the above arguments, it seems most likely that Leo I must be a member of a higher mass concentration and cannot be bound to the MW alone. We therefore discard Leo I from further analysis. 


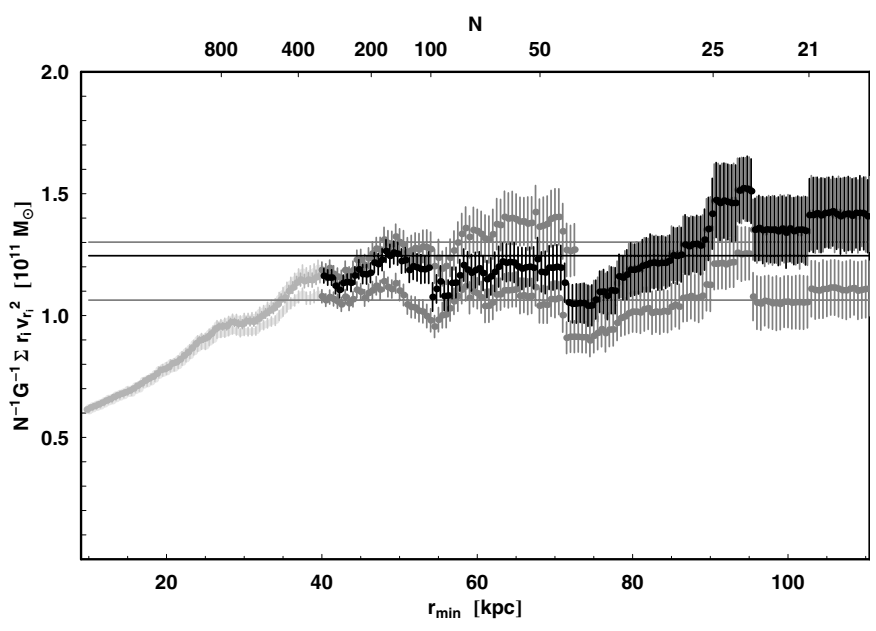

Fig. 2. Mean values $\frac{1}{N G} \sum_{i} r_{i} v_{r, i}^{2} ; r_{i}>r_{\min }, N=\sum_{i} 1$; for tracers outside the spherical surface of radius $r_{\min }$ shown as a function of that radius (the vertical bars represent the standard deviation for random subsets with $7 / 8$ of the elements). The curve in the top (top, gray) (the SI sample) discards only Leo I; the curve in the middle (middle, black) discards an additional single tracer J160826.42+065542.3 with $\frac{1}{2 G} r v_{r}^{2}>5.0 \times 10^{11} M_{\odot}$; the curve in the bottom (bottom, gray) (the SII sample), in addition to the previous two tracers, discards 4 more tracers (88-TARG37, Hercules, J234809.03-010737.6, and J124721.34+384157.9) with $\frac{1}{2 G} r v_{r}^{2} \geq 3.49 \times 10^{11} M_{\odot}$. The two lower curves are approximately flat in a wide range of radii $40 \mathrm{kpc}<r_{\min }<$ $90 \mathrm{kpc}$. The thin straight lines show the mean values in that region. It is important to note that the resulting mass estimate for sample SI compared to that for sample SII is increased by only a factor of $\approx 1.16$, which is comparable to, or even less than, model errors for the MW mass in the literature.

\subsection{Two samples of tracers}

When the MW's mass is expected to be lower, as suggested by the discussion in Sect. 1, some of the tracers cannot be gravitationally bound to it and, therefore, similar to Leo I, should not be included in preparing the RVD profile. The following criterion can be used for hypothesizing which of satellites might be unbound. The simple calculation of Sect. 1 leads to the mass estimator $\widetilde{M}_{r}=\frac{\mu}{N_{r}} \sum_{r_{i}<r} r_{i} v_{r, i}^{2}$ with $\mu=4$ for $\beta \approx 0$ (or $\beta \approx \epsilon / 2$ with a small $\epsilon>0$ ), where the summation is taken over a number $N_{r}$ of objects with the radial distance not lower than $r$. This result follows at once for an approximately flat $r\left\langle v_{r}^{2}\right\rangle$, when one can use $\left\langle r v_{r}^{2}\right\rangle$ instead, but it can also be arrived at without this reservation by applying Eq. (23) given in Watkins et al. (2010) with suitable parameters. As can be seen in Fig. 2, the mass estimate $\widetilde{M} \approx 4-5 \times 10^{11} M_{\odot}(\mu \approx 4)$ is approximately invariable in the region from $40 \mathrm{kpc}$ to $90 \mathrm{kpc}$, consistently with what one would expect for a compact concentration of mass. (Beyond $90 \mathrm{kpc}$ the statistics is too small.) This is in accord with the value $3.5-5 \times 10^{11} M_{\odot}$ within $150 \mathrm{kpc}$, obtained in a Keplerian halo model (Deason et al. 2012b). With the limiting value $\mu=2$ for $\beta=1, \widetilde{M}$ might be as low as $2.1-2.6 \times 10^{11} M_{\odot}$ (see, the straight lines in Fig. 2). The two estimates suggest that satellites with $\frac{1}{2 G} r v_{r}^{2}$ greater than $5 \times 10^{11} M_{\odot}$ or $3 \times 10^{11} M_{\odot}$, respectively, might not be bound to the MW and should be excluded prior to preparing the RVD profile. We further assume these two possibilities by considering two samples of tracers SI and SII as specified in Table 1.
Table 1. Radial velocity tracers with the largest $\frac{r v_{r}^{2}}{2 G}$ and samples SI and SIl used in the text.

\begin{tabular}{|c|c|c|c|c|}
\hline $\begin{array}{l}\text { Tracer's } \\
\text { name }\end{array}$ & $\begin{array}{l}\text { Short } \\
\text { name }\end{array}$ & $\begin{array}{c}r v_{r}^{2} / 2 G \\
{\left[10^{11} M_{\odot}\right]}\end{array}$ & $\begin{array}{l}\text { Distance } \\
][\mathrm{kpc}]\end{array}$ & SISII \\
\hline Leo I & & 8.29 & 254 & -- \\
\hline $\mathrm{J} 160826.42+065542.3$ & $x$ & 5.62 & 72.7 & -- \\
\hline $\begin{array}{l}\text { 88-TARG37 } \\
\text { Hercules } \\
\text { J234809.03-010737.6 } \\
\text { J124721.34+384157.9 }\end{array}$ & $\begin{array}{l}\text { A } \\
B \\
C \\
D\end{array}$ & $\begin{array}{l}4.00 \\
3.65 \\
3.51 \\
3.49 \\
\end{array}$ & $\begin{array}{c}55.3 \\
132 \\
54.2 \\
41.5 \\
\end{array}$ & $\begin{array}{l}+- \\
+- \\
+- \\
+-\end{array}$ \\
\hline $\begin{array}{l}\text { J232526.89-094433.5 } \\
\text { J121222.29+422502.0 } \\
\text { J115555.42+365908.6 } \\
\text { J090016.24+341342.4 } \\
\text { J144258.28+575339.3 } \\
\text { Eridanus } \\
\text { J160507.08+293222.3 } \\
\text { Pal14 } \\
\text { 85-TARG17 }\end{array}$ & & $\begin{array}{l}2.88 \\
2.84 \\
2.77 \\
2.61 \\
2.57 \\
2.56 \\
2.54 \\
2.52 \\
2.34\end{array}$ & $\begin{array}{l}53.8 \\
37.7 \\
51.0 \\
48.8 \\
48.3 \\
95.4 \\
42.2 \\
71.3 \\
45.1\end{array}$ & $\begin{array}{l}++ \\
++ \\
++ \\
++ \\
++ \\
++ \\
++ \\
++ \\
++\end{array}$ \\
\hline$\vdots$ & & $\vdots$ & $\vdots$ & \\
\hline
\end{tabular}

Notes. The numerical values were calculated in the Galactocentric coordinate system, assuming $V_{\mathrm{o}}=240 \mathrm{~km} \mathrm{~s}^{-1}, R_{\mathrm{o}}=8.5 \mathrm{kpc}, U=$ $11.1 \mathrm{~km} \mathrm{~s}^{-1}, V=12.24 \mathrm{~km} \mathrm{~s}^{-1}$, and $W=7.25 \mathrm{~km} \mathrm{~s}^{-1}$.

\subsection{The radial velocity dispersion profile}

As explained in Sect. 2.2, we consider two RVD profiles based on tracers from samples SI and SII (see Table 1). The PDFs are shown in Fig. 3 and also compared in Fig. 4, in the background of all tracers.

The RVD curves in Fig. 3 were obtained as follows: for any $r_{i}$ all of $n_{i}$ tracers are taken inside a spherical shell $\left|r-r_{i}\right|<w / 2$ of a fixed width $w$. When necessary, $w$ is increased so that $n_{i}$ is never lower than some fixed $n$, which effectively increases the bin size at large radii where the statistics are poor because there were few tracers. Then $m$ random subsets of size $\frac{2}{3} n_{i}$ are chosen, and a quartet of numbers is assigned to each of them: two mean values $\langle r\rangle$ and $G^{-1}\left\langle r v_{r}^{2}\right\rangle$, and two numbers $r_{\min }$ and $r_{\max }$, the smallest and the largest $r$ in each subset. This is repeated for all $r_{i}$ at various $n$. Then an ordered list consisting of all the quartets is formed, sorted according to increasing $\langle r\rangle$. Finally, a moving average is performed over quartets with $\langle r\rangle$ falling in a window of width $W$, when the window moves through the entire range of $r$. In effect, a curve is obtained on the plane $\left(r, G^{-1} r v_{r}^{2}\right)$. We assumed $w=9 \mathrm{kpc}, m=89, W=6 \mathrm{kpc}$, and $n$ from 15 to 22. For each $r$ the horizontal "error bars" represent the intervals $\left\langle r_{\min }\right\rangle<r<\left\langle r_{\max }\right\rangle$, while the vertical "error bars" represent the standard deviations of $G^{-1}\left\langle r v_{r}^{2}\right\rangle$ times $\sqrt{3}$, and measure a Monte Carlo-like estimate of the uncertainty in $G^{-1}\left\langle r v_{r}^{2}\right\rangle$ due to various subsets of tracers taken in obtaining the mean values $\left\langle r v_{r}^{2}\right\rangle$.

It can be seen that the RVD profiles largely depend on two tracers with the highest $\frac{1}{2 G} r v_{r}^{2}, \mathrm{~B}$, and $\mathrm{X}$, while the inclusion/exclusion of the other three tracers $A, C$, and $D$, is not so decisive. Tracer $X$ certainly cannot be bound to the MW due to the recent low-mass estimates referred to earlier and therefore should not be included. This consideration substantiates our choice of the two basic RVD profiles.

The RVD profiles in Fig. 3 can be interpolated to form continuous curves that can be regarded as smooth model curves, consistent with the measurements within certain uncertainty 

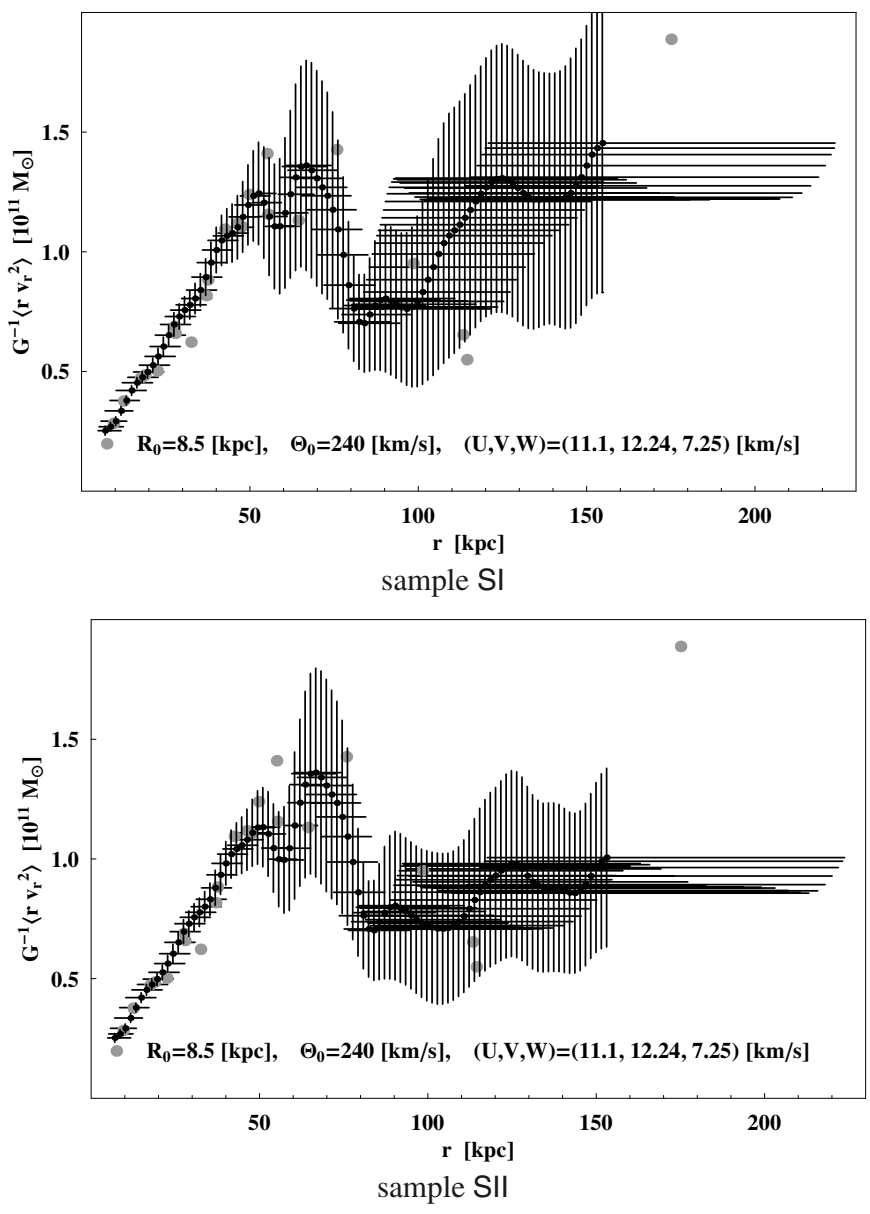

Fig. 3. Radial velocity dispersion (RVD) profiles $G^{-1}\left\langle r v_{r}^{2}\right\rangle$ for tracers with $\frac{1}{2 G} r v_{r}^{2}<5.0 \times 10^{11} M_{\odot}$ (the SI sample, top panel); and $\frac{1}{2 G} r v_{r}^{2}<3.49 \times 10^{11} M_{\odot}$ (the SII sample, bottom panel). The horizontal bars represent the effective radial bin size of the moving average. The vertical bars indicate the spread in the RVD due to the inclusion/exclusion of random subsets of tracers. A detailed description of how these profiles were obtained can be found in Sect. 2.3. As reference values, we show $G^{-1} r\left\langle v_{r}^{2}\right\rangle$ calculated based on the RVD points in Xue et al. (2008) and Deason et al. (2012b) (large gray circles). Note that Leo I was excluded from our analysis. See also the comparison of the profiles in Fig. 4.

limits ${ }^{6}$. With the aid of the method introduced in Sect. 3.1.3, a number of PDFs can be found, giving rise to the respective theoretical RVD curves consistent with the smooth model curves within the same limits. When this is possible with some mass $M$, we say that the $M$, as an estimate for the MW mass, accounts for the radial motions of tracers.

\section{Keplerian ensemble method}

To estimate the lower bound for the MW mass, we assume that external tracers move as test bodies in the host gravitational field of a compact mass distribution. In this case, at sufficiently

\footnotetext{
6 If need be, one can choose a subset of points on these curves with the respective "error bars" akin to the RVD data consisting of few points as usually given in the literature, such that the difference between the abscissas of the neighboring points are determined by the extension of the respective horizontal bars (e.g., a half of the mean of the extension). There are many such subsets, depending on the position of the initial point; however, all these subsets will be consistent with measurements within "errors".
}

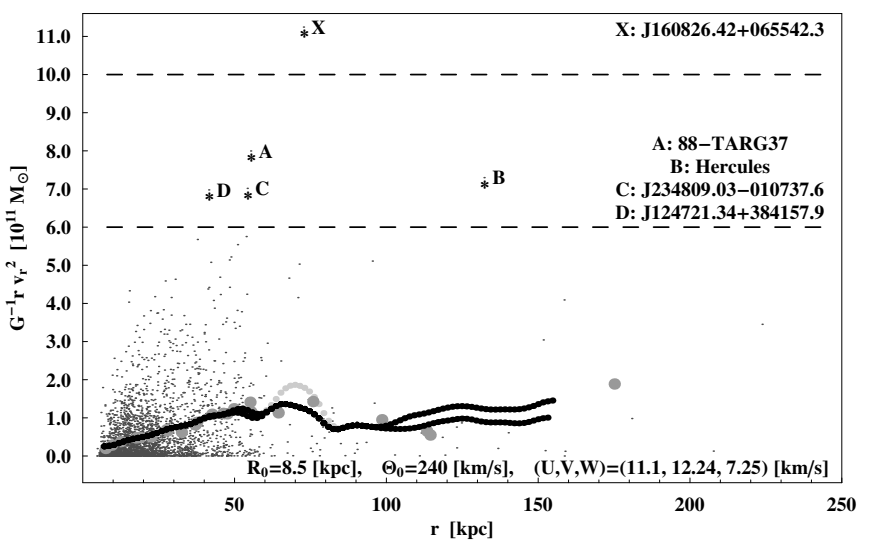

Fig. 4. Radial velocity tracers (gray dots/black stars) and the radial velocity dispersion (RVD) profiles (black circles) of Fig. 3 shown on the $\left(r, G^{-1} r v_{r}^{2}\right)$ plane. The RVDs are curves prepared assuming that either only Leo I and X (the SI sample) or both these two and A, B, C, D (the SIl sample), are excluded (see Table 1). With all tracers included (with the exception of Leo I), a bump structure centered at $\approx 70 \mathrm{kpc}$ would appear (light gray circles). The inclusion or exclusion of $A, C$, and $D$ does not influence the RVDs significantly (only a little bit close to $\approx 50 \mathrm{kpc}$ ), whereas the inclusion of $\mathrm{B}$ rises the RVD curve for radii greater than $100 \mathrm{kpc}$. Thus, the RVD curves are mainly dependent on only two tracers B (Hercules) and X. The large gray circles are the reference $G^{-1} r\left\langle v_{r}^{2}\right\rangle$ values based on the literature (the same as in Fig. 3).

large radii, a contribution from the higher multipoles of the field should be small compared to the monopole part, and the motion of distant test bodies should be approximately describable in terms of Keplerian orbits. Because point mass formulae can be used as order-of-magnitude estimates of gravitating mass, the compactness assumption does not necessarily reject the possibility that an extended massive dark matter halo may be present. Also, the compactness assumption does not a priori reject the possibility that the spatially extended dark matter halo component may be absent. The latter hypothesis should be tested in accordance with Occam's razor rule, before one decides to introduce new forms of matter, such as a ubiquitous invisible substance consisting of dark matter particles of unknown nature and, so far, eluding detection in the laboratory by producing no observable non-gravitational effects (e.g., recent null results, LUX Collaboration 2013).

\subsection{The method}

The motion of a test body in a spherically symmetric potential $\Phi(r)$ is flat. It occurs in a plane through the center of symmetry. The plane is determined by two integrals of motion that fix the unit vector normal to the plane. The additional two integrals are energy $E$ and the magnitude of angular momentum $J$ (both per unit mass). In terms of angular velocity $\omega, \omega^{2}=\dot{\theta}^{2}+\dot{\phi}^{2} \sin ^{2} \theta$, they read as $r^{2} \omega^{2}=\frac{J^{2}}{r^{2}}$ and $v_{r}^{2}=2 E-2 \Phi-\frac{J^{2}}{r^{2}}$. In the special case of Newtonian potential $\Phi=-\frac{G M}{r}$, there is an additional integral of motion indicating a fixed direction in the plane of motion.

The condition $v_{r}^{2} \geq 0$ for all $r>0$ with $J \neq 0$ gives $1+\frac{2 E J^{2}}{G^{2} M^{2}} \geq 0$. We are only interested in spatially bound orbits. Two turning points (where $v_{r}=0$ ) will be possible for $E<0$ and $1+\frac{2 E J^{2}}{G^{2} M^{2}}<1$. Elliptical orbits are therefore possible only for pairs $(E, J)$ such that $0 \leq 1+\frac{2 E J^{2}}{G^{2} M^{2}}<1$. Elliptical motion can be thus uniquely determined by specifying five numbers: three Euler angles describing orientation of the orbit in space, and a 
pair of numbers $(e, \epsilon)$ defined by $e^{2}=1+\frac{2 E J^{2}}{G^{2} M^{2}}, 0 \leq e<1$, and $\epsilon=-\frac{R E}{G M}>0$. Here, $R$ is an arbitrary unit of length, $e$ the eccentricity, and $\epsilon$ a measure of energy determining the length of the large semi-axis, which is $\frac{R}{2 \epsilon}$. The turning points are $\frac{R}{2 \epsilon}(1 \pm e)$. The dimensionless parameters $(e, \epsilon)$ play the central role in further considerations.

To find various expectation values for a spherically symmetric collection of confocal ellipses (called a Keplerian ensemble), it suffices to know a PDF that describes the number of ellipses with various $e$ and $\epsilon$. It will be related to the distribution function $f(\boldsymbol{r}, \boldsymbol{v})$ in $\mu$-phase-space. We assume that $f$ can be expressed through first integrals $e$ and $\epsilon$. Then, $f$ is stationary and satisfies the necessary condition $\partial_{t} f+\partial_{q^{i}} f+\partial_{\dot{q}^{i}} f \partial^{i} \Phi=0$ for a collisionless system. This is the wording of the Jeans theorem (Jeans 1915) in our situation. In general, the theorem states that for a system in steady state, the PDF is a function of isolating integrals of motion (Jeans 1915). (Under spherical symmetry two such integrals suffice.)

\subsubsection{A cutoff phase-space and the resulting expectation values}

The integrals of motion $e$ and $\epsilon$ can be regarded as new, independent phase variables. By making a transformation from ordinary spherical coordinates $r, \theta, \phi, v_{r}, v_{\theta}, v_{\phi}$ to new coordinates $u, \theta, \phi, \epsilon, e, \psi$ of the form $r \rightarrow R u$, $(\theta, \phi) \rightarrow(\theta, \phi), v_{r}^{2} \rightarrow \frac{G M}{R}\left(\frac{2}{u}-\frac{1-e^{2}}{2 \epsilon} \frac{1}{u^{2}}-2 \epsilon\right)$, and $\left(v_{\theta}, v_{\phi}\right) \rightarrow$ $\sqrt{\frac{G M}{R}} u^{-1}\left(\frac{1-e^{2}}{2 \epsilon}\right)^{1 / 2}(\sin \psi, \cos \psi)$, the original volume element $r^{2} \mathrm{~d} r \sin \theta \mathrm{d} \theta \mathrm{d} \phi \frac{\mathrm{d} v_{r}^{2}}{2 v_{r}} \mathrm{~d} v_{\theta} \mathrm{d} v_{\phi}$ is transformed (up to a constant factor) to $\mathcal{J}(e, \epsilon, u) \mathrm{d} u \sin \theta \mathrm{d} \theta \mathrm{d} \phi \mathrm{d} \psi \mathrm{d} \epsilon e \mathrm{~d} e$, with

$\mathcal{J}(e, \epsilon, u)=\left[\epsilon\left(\epsilon-\frac{1-e}{2 u}\right)\left(\frac{1+e}{2 u}-\epsilon\right)\right]^{-1 / 2}$.

Since we assume spherical symmetry, the angles $\phi, \theta$, and $\psi$ can be integrated out. The remaining part of the integration domain is determined by the function $\mathcal{J}$. Furthermore, in finding the expectation values as functions of $r$, the integration must be taken over all ellipses that intersect a spherical thin shell of a fixed radius $r$, concentric to the center of symmetry. Given $0<r<\infty$ and $0 \leq e<1$, we have $\frac{1-e}{2 u} \leq \epsilon \leq \frac{1+e}{2 u}$ for such orbits. Hence, we arrive at the distribution integral

$\int_{0}^{\infty} \mathrm{d} u \int_{0}^{1} e \mathrm{~d} e \int_{\frac{1-e}{2 u}}^{\frac{1+e}{2 u}} \mathrm{~d} \epsilon \mathcal{J}(e, \epsilon, u) f(e, \epsilon)$,

which equals $\int f(\boldsymbol{r}, \boldsymbol{v}) \mathrm{d}^{3} \boldsymbol{r} \mathrm{d}^{3} \boldsymbol{v}$ to within a constant factor.

Next, for the physical reasons, we assume all orbits of the ensemble to be contained entirely within a spherical shell $R u_{\mathrm{a}}<$ $r<R u_{\mathrm{b}}$, that is, in between two boundary spheres of radii $r_{\mathrm{a}}=R u_{\mathrm{a}}$ and $r_{\mathrm{b}}=R u_{\mathrm{b}}$. (As a byproduct, the normalized cumulative number of objects will be automatically integrable.) This spatial boundary imposes additional restrictions on the integration domain in the phase-space, changing considerably the support of $f(\epsilon, e)$. Then, $u_{\mathrm{a}}<\frac{1-e}{2 \epsilon}$ and $\frac{1+e}{2 \epsilon}<u_{\mathrm{b}}$. Hence, $\frac{1+e}{2 u_{\mathrm{b}}}<\epsilon<\frac{1-e}{2 u_{\mathrm{a}}}$ and also $0 \leq e<\frac{u_{\mathrm{b}}-u_{\mathrm{a}}}{u_{\mathrm{b}}+u_{\mathrm{a}}}<1$. As a result, the integration domain in the integral (2) gets shrunk to a quadrilateral abcB, as shown in Fig. 5. However, the integration requires splitting the $u$-dependent domain into three parts. To overcome this difficulty we introduce a mapping $(\alpha, \beta) \rightarrow\left(e(\alpha, \beta), \epsilon_{u}(\alpha, \beta)\right)$ from a rectilinear region with cartesian coordinates $(\alpha, \beta)$ to a $u$-dependent region abcB. This leads to the following coordinate

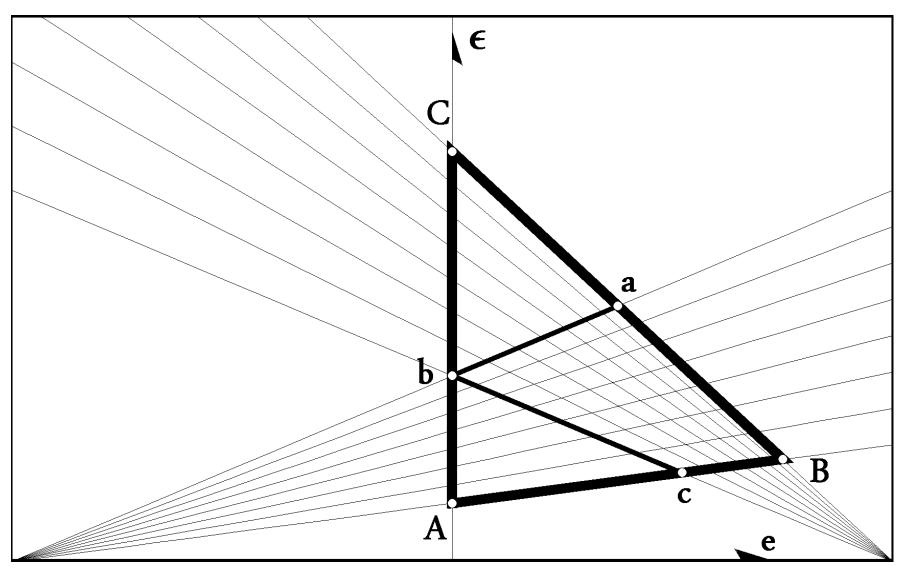

Fig. 5. Each point on the $(e, \epsilon)$ plane represents an elliptical orbit with eccentricity $e$ and energy $-\epsilon$. The ABC triangle with vertices $\mathrm{A}\left(0, \frac{1}{2 u_{\mathrm{b}}}\right)$, $\mathrm{B}\left(\frac{u_{\mathrm{b}}-u_{\mathrm{a}}}{u_{\mathrm{b}}+u_{\mathrm{a}}}, \frac{1}{u_{\mathrm{a}}+u_{\mathrm{b}}}\right), \mathrm{C}\left(0, \frac{1}{2 u_{\mathrm{a}}}\right)$ is a locus of all confocal ellipses lying entirely in-between two bounding spheres of fixed radii $u_{\mathrm{a}}$ and $u_{\mathrm{b}}$ and centered on the focal point. The two families of mutually crossing lines $\epsilon=\frac{1+e}{2 u} \alpha$ through point $(-1,0)$ and $\epsilon=\frac{1-e}{2 u} \beta$ through point $(1,0)$ give rise to a new coordinate system $(\alpha, \beta)$ on the $(e, \epsilon)$ plane. When $\alpha \in\left(\frac{u}{u_{\mathrm{b}}}, 1\right)$ and $\beta \in\left(1, \frac{u}{u_{\mathrm{a}}}\right)$ the coordinates cover a $u$-dependent quadrilateral integration domain abcB with vertices a $\left(\frac{u-u_{\mathrm{a}}}{u+u_{\mathrm{a}}}, \frac{1}{u+u_{\mathrm{a}}}\right), \mathrm{b}\left(0, \frac{1}{2 u}\right)$, $\mathrm{c}\left(\frac{u_{\mathrm{b}}-u}{u_{\mathrm{b}}+u}, \frac{1}{u_{\mathrm{b}}+u}\right)$, and $\mathrm{B}$, which is the locus of all orbits $(e, \epsilon)$ crossing at least once a sphere of a given radius $u_{\mathrm{a}}<u<u_{\mathrm{b}}$.

change in the reduced phase space $(\epsilon, e, u)$ in the above distribution integral:

$$
\begin{gathered}
e \rightarrow e(\alpha, \beta)=\frac{\beta-\alpha}{\beta+\alpha}, \quad \epsilon \rightarrow \epsilon(u, \alpha, \beta)=\frac{1}{u} \frac{\alpha \beta}{\alpha+\beta}, \quad u \rightarrow u, \\
\frac{u}{u_{\mathrm{b}}}<\alpha<1, \quad 1<\beta<\frac{u}{u_{\mathrm{a}}}, \quad 0<u_{\mathrm{a}}<u<u_{\mathrm{b}} .
\end{gathered}
$$

The interpretation and the origin of this coordinate change is shown in Fig. 5. We finally get

$$
\begin{aligned}
& \int f(\boldsymbol{r}, \boldsymbol{v}) \mathrm{d}^{3} \boldsymbol{r} \mathrm{d}^{3} \boldsymbol{v} \propto \int_{u_{\mathrm{a}}}^{u_{\mathrm{b}}} \mathrm{d} u \sqrt{u} \mu_{u}[f], \quad \text { where } \\
& \mu_{u}[f]=\int_{u / u_{\mathrm{b}}}^{1} \frac{\mathrm{d} \alpha}{\sqrt{1-\alpha}} \int_{1}^{u / u_{\mathrm{a}}} \frac{\mathrm{d} \beta}{\sqrt{\beta-1}} \frac{\beta-\alpha}{(\alpha+\beta)^{5 / 2}} f\left(\frac{\beta-\alpha}{\beta+\alpha}, \frac{1}{u} \frac{\alpha \beta}{\alpha+\beta}\right) .
\end{aligned}
$$

Now, given a $f(e, \epsilon)$, all expectation values can be determined in principle. In calculating them, the following expressions are useful:

$$
\frac{r v_{r}^{2}}{2 G M}=\frac{(1-\alpha)(\beta-1)}{\alpha+\beta}, \quad \frac{r v_{\phi}^{2}}{G M}=\frac{2}{\alpha+\beta}, \quad e=\frac{\beta-\alpha}{\beta+\alpha} .
$$

For the purpose of further applications, the averages over concentric thin spherical shells are important. The mean value of a function $g$ defined on a spherical shell of radius $r$, and consequently, the average over all spherical shells can be calculated from

$\langle g\rangle_{r}=\frac{\mu_{u}[f g]}{\mu_{u}[f]} \quad$ and $\quad\langle g\rangle=\frac{\int \mathrm{d} u \sqrt{u}\langle g\rangle_{u} \mu_{u}[f]}{\int \mathrm{d} u \sqrt{u} \mu_{u}[f]}$,

respectively. The expression for $\langle g\rangle_{r}$ is quite analogous to a conditional probability "A provided that B" for events A and B. 


\subsubsection{An orthogonal decomposition of the phase-space function}

The problem of finding a PDF, $f(e, \epsilon)$, on a triangular domain (Fig. 5) can be reduced to finding a series expansion of an auxiliary function $h(\xi(e, \epsilon), \eta(e, \epsilon))$ in a basis of polynomials orthonormal on a simplex in the plane $\xi, \eta$ (we assume that $f \equiv h^{2}$, then $f$ is non-negative; the transformation functions $\xi(e, \epsilon), \eta(e, \epsilon)$ still need to be specified). To this end we apply the Gram-Schmidt method with a scalar product $g(u, v)=$ $2 \int_{0}^{1} \mathrm{~d} \xi \int_{0}^{1-\xi} \mathrm{d} \eta u(\xi, \eta) v(\xi, \eta)$ on the unit simplex $0<\xi<1$, $0<\eta<1, \xi+\eta<1$. First, we define two families of polynomials of degree that does not exceed a given $d>0$, namely, $A_{n, k}: \eta^{k} \xi^{n-k}-\xi^{k} \eta^{n-k}$ and $S_{n, k}: \eta^{k} \xi^{n-k}+\xi^{k} \eta^{n-k}$, with $0 \leq n \leq d$, $0 \leq k \leq n$. By symmetry, any $A$ is orthogonal to any $S$. Then, we sort polynomials $S$, to get a sequence with a nondecreasing degree and take their union, thereby obtaining a reduced sequence $S^{\prime}$. We transform $S^{\prime}$ to another sequence by the consecutive projections $s_{m}^{\prime}=S_{m}^{\prime}-\sum_{i=1}^{m-1} \frac{g\left(S_{m}^{\prime}, S_{i}^{\prime}\right)}{g\left(S_{i}^{\prime}, S_{i}^{\prime}\right)} S_{i}^{\prime}$ and normalize $s_{m}^{\prime} \rightarrow s_{m}=\left(g\left(s_{m}^{\prime}, s_{m}^{\prime}\right)\right)^{-1 / 2} s_{m}^{\prime}$. We repeat the same procedure for nonzero polynomials $A$ to obtain a sequence $a_{m^{\prime}}$. Finally, we take the union of sequences $s_{m}$ and $a_{m^{\prime}}$, and sort with respect to the increasing degree, obtaining the required basic polynomials $\mathcal{P}_{j}(\xi, \eta), j=0,1, \ldots,(d+1)(d+2) / 2$. The initial 45 polynomials obtained this way are shown in Fig. 6. Finally, we make a coordinate change $\xi=\frac{u_{\mathrm{b}}+u_{\mathrm{a}}}{u_{\mathrm{b}}-u_{\mathrm{a}}} e, \eta=\frac{u_{\mathrm{a}}}{u_{\mathrm{b}}-u_{\mathrm{a}}}\left(2 u_{\mathrm{b}} \epsilon-e-1\right)$, thereby mapping the simplex to the triangular domain ABC in Fig. 5. This way, the task of finding an orthonormal basis, of $D_{d}=(d+1)(d+$ 2) $/ 2$ polynomials $Q_{j}(e, \epsilon)=\mathcal{P}_{j}\left(\frac{u_{\mathrm{b}}+u_{\mathrm{a}}}{u_{\mathrm{b}}-u_{\mathrm{a}}} e, \frac{u_{\mathrm{a}}}{u_{\mathrm{b}}-u_{\mathrm{a}}}\left(2 u_{\mathrm{b}} \epsilon-e-1\right)\right)$ of degree not exceeding $d$ in $e$ and $\epsilon$, has been completed.

\subsubsection{Finding the phase-space function and the derived quantities}

The theoretical RVD can be compared with the quantity $\left\langle r v_{r}^{2}\right\rangle$ determined based on measurements for a large enough sample of points $r_{i}$. Given $d$ and mass $M$, this comparison enables us to find a set of optimal coefficients in the following expansion of the phase-space function $f(e, \epsilon)$ :

$$
f(e, \epsilon)=h^{2}(e, \epsilon), \quad h(e, \epsilon) \approx \sum_{k=1}^{D_{d}} h_{k} Q_{k}(e, \epsilon)
$$

by minimizing the following mismatch function: ${ }^{7}$

$$
\delta_{M}=\sqrt{\frac{\frac{1}{N} \sum_{i}\left(\bar{\sigma}\left(u_{i}\right)-\left\langle\frac{r_{i} v_{r}^{2}\left(r_{i}\right)}{G M}\right\rangle\right)^{2}}{\left(\frac{1}{N} \sum_{i}\left\langle\left\langle\frac{r_{i} v_{r}^{2}\left(r_{i}\right)}{G M}\right\rangle\right)^{2}\right.}}, \quad u_{i}=r_{i} / R,
$$

$N=\sum_{i} 1$ (it suffices that $N$ be several times greater than $D_{d}$ ), where

$$
\bar{\sigma}(u)=\frac{\sum_{k, l}^{D_{d}} h_{k} h_{l} \mu_{u}\left[\sigma \mathcal{P}_{k} \mathcal{P}_{l}\right]}{\sum_{k, l}^{D_{d}} h_{k} h_{l} \mu_{u}\left[\mathcal{P}_{k} \mathcal{P}_{l}\right]}, \quad \sigma=\frac{2(1-\alpha)(\beta-1)}{\alpha+\beta},
$$

with $\frac{\beta-\alpha}{\beta+\alpha}$ and $\frac{1}{u} \frac{\alpha \beta}{\alpha+\beta}$ substituted in $\mathcal{P}_{\mathrm{S}}$ for $e$ and $\epsilon$, respectively. The integrals $\mu_{u}\left[\mathcal{P}_{k} \mathcal{P}_{l}\right]$ and $\mu_{u}\left[\sigma \mathcal{P}_{k} \mathcal{P}_{l}\right]$ defining functions of a

\footnotetext{
7 To measure the accuracy of a fit $Y(x)$ to data points $\left(x_{i}, y_{i}\right)$, we use a dimensionless number $\delta$ such that $\delta^{2}=\frac{1}{N \bar{y}^{2}} \sum_{i}\left(Y\left(x_{i}\right)-y_{i}\right)^{2}$, where $\bar{y}=\frac{1}{N} \sum_{i}\left|y_{i}\right|$. The definition of $\delta_{M}$ agrees with that of $\delta$.
}

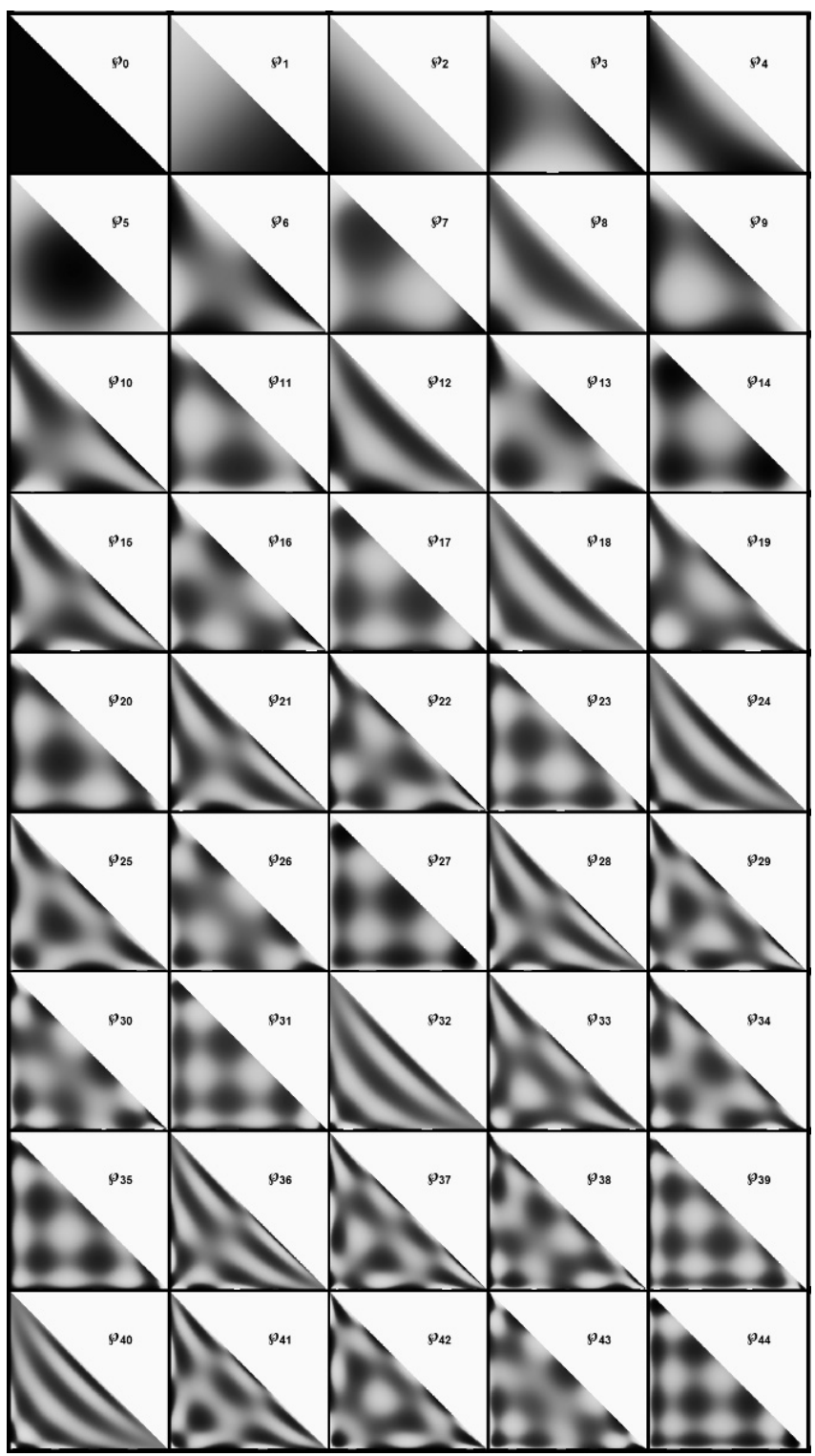

Fig. 6. Contour maps (with a certain automatic shading function) of 45 initial polynomials from a basis of polynomials orthonormal with respect to a standard scalar product on the unit simplex $\xi+\eta-1<0$, $0<\xi<1,0<\eta<1$, constructed using the procedure of Sect. 3.1.2

single argument $u$ leads to integrals of the general form:

$\frac{1}{u^{s}} \int_{u / u_{\mathrm{b}}}^{1} \mathrm{~d} \alpha \int_{1}^{u / u_{\mathrm{a}}} \mathrm{d} \beta \frac{\alpha^{p} \beta^{q}}{(\alpha+\beta)^{r} \sqrt{(1-\alpha)(\beta-1)(\alpha+\beta)}}$,

with integers $p, q, r, s$. The analytical form of this integral can be found recursively for any set of integers $p, q, r{ }^{8}$

Since $\delta_{M}$ is homogenous with degree 1 in the variables $h_{k}$, it only depends on points on a $\left(D_{d}-1\right)$-dimensional unit sphere. To

\footnotetext{
8 We have not found any general formula for such an integral; however, having written a code using a computer algebra system, we were able to find the integrals in analytic form by recursions for any $p, q, r$. The alternative way - a numerical integration - would be inefficient in the minimization procedure; we observed that either the errors accumulated in an uncontrolled fashion, resulting in a high noise level in $\delta_{M}$, or the computation was too time-consuming when the precision was increased to keep the errors within acceptable bounds.
} 


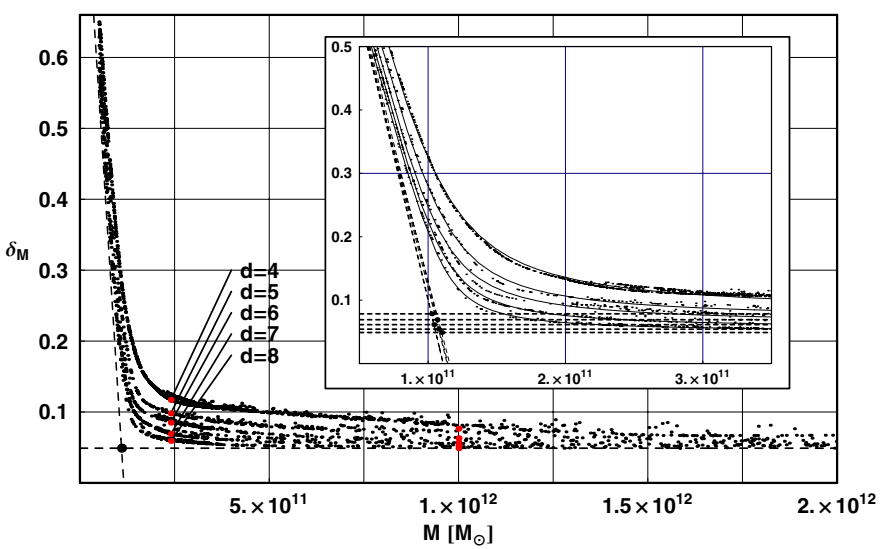

Fig. 7. A $\chi^{2}$-like test defined by $\delta_{M}$ for best-fit curves to the RVD profile of sample SII (see Fig. 3, bottom panel) shown as a function of the central mass $M$. The phase-space support used to obtain these results was fixed by $u_{\mathrm{a}}=18 \mathrm{kpc}$ and $u_{\mathrm{b}}=300 \mathrm{kpc}$, and the fitting region was limited to $r>22 \mathrm{kpc}$. One can distinguish 5 accumulation curves that correspond to various degrees $d$ of polynomials used to approximate the phase distribution function (here, $d=4,5,6,7,8$ ). These curves were compared with best-fitting hyperbolas in the inset panel. Abscissae of crossing points of the corresponding asymptotes suggest the presence of a lower bound for mass in the limit of large $d$. The red dots represent minimum values of $\delta_{M}$ found at $M=2.4 \times 10^{11} M_{\odot}$ and, to compare with, at $M=1.0 \times 10^{12} M_{\odot}$ (for $d=8$ points with $u_{\mathrm{b}}=240 \mathrm{kpc}$ were also included).

find the $\delta_{M}$, one starts with a random point on that sphere (with the help of a generator uniform on that sphere), and then the minimization procedure is run. There can be many such minima, or a minimum can be degenerated. Having found $h_{k}$ with this method, secondary quantities other than $\bar{\sigma}(u)$ can be computed, using definitions like (4). They lead to expressions $\bar{e}(u), \bar{\beta}(u)$, etc., of the same general form as that of $\bar{\sigma}(u)$ in (8) (with quantities of the kind as in (4) substituted for $\sigma$ in $\left.\mu_{u}\left[\sigma \mathcal{P}_{k} \mathcal{P}_{l}\right]\right)$, that is, a quotient of two quadratic forms in $D_{d}$ variables $h_{i}$.

\section{Results}

Using the minimization method of Sect. 3.1.3, we started by generating several best fit curves to the RVD profile of the SII sample of tracers and assumed various central masses $M$, ranging from $^{9} 0.5 \times 10^{11} M_{\odot}$ to $50 \times 10^{11} M_{\odot}$. This allowed us to compare the quality of best fits measured by the mismatch function $\delta_{M}$ defined in (7) and to see how it changes with the number $D_{d}$ of polynomials used in (6) to approximate the PDF. The result of this comparison is presented in Fig. 7, where $\delta_{M}$ is shown versus the central mass $M$ for various $d$. The example best-fit curves to the RVD profile (assuming $d=8$ ) were compared in Fig. 8. Lower panels in Fig. 8 show the corresponding secondary quantities. As expected, they can change significantly when RVD best-fit curves are not changed at all. This illustrates our earlier theoretical expectation that $M$ cannot be determined based on the measurements of the radial motions alone. Also, due to the flattening of the $\delta_{M}$ curves in Fig. 7 for high masses, the optimum mass, chosen to correspond to the lowest $\delta_{M}$ fit (a global minimum), would have unacceptably large uncertainty (as would the secondary quantities that are largely $M$-dependent). Thus, the criterion of mass must be different. Nevertheless, one can estimate a lower bound for $M$.

\footnotetext{
9 Most of the values in this range of masses are physically implausible and were only used for illustration.
}
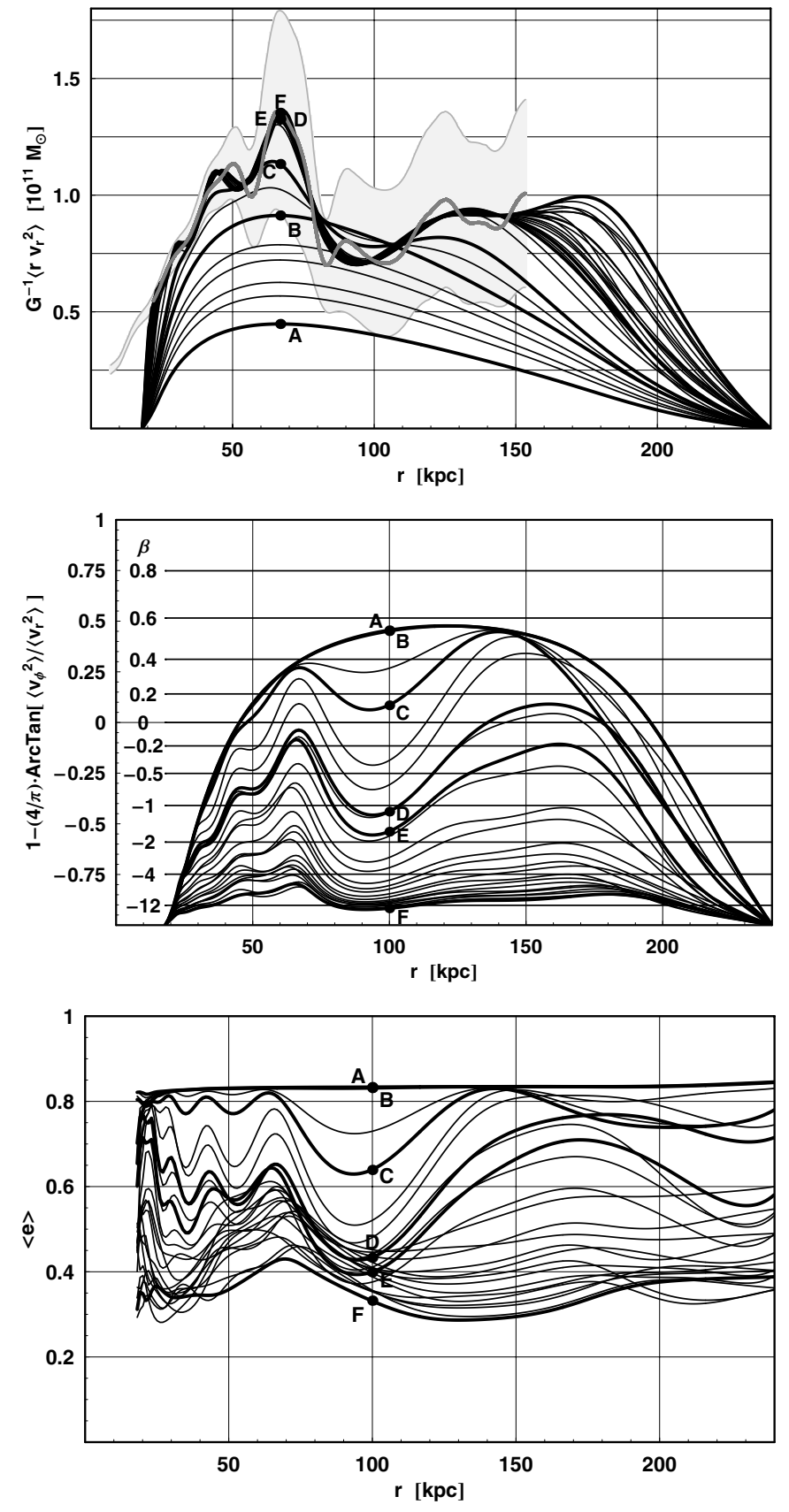

Fig. 8. Top: example best-fit curves to the RVD profile of sample SII for masses ranging from $0.5 \times 10^{11} M_{\odot}$ to $10 \times 10^{11} M_{\odot}\left(\mathrm{A}: 0.5 \times 10^{11} M_{\odot}\right.$, B: $1.02 \times 10^{11} M_{\odot}, \mathrm{C}: 1.3 \times 10^{11} M_{\odot}$, D: $2.09 \times 10^{11} M_{\odot}$, E: $2.4 \times 10^{11} M_{\odot}$, F: $\left.10.2 \times 10^{11} M_{\odot}\right)$, assuming $d=8$ and $u_{\mathrm{a}}=18 \mathrm{kpc}, u_{\mathrm{b}}=240 \mathrm{kpc}$ $(\Rightarrow 0<e<0.83)$. The light gray region is the uncertainty in the RVD profile corresponding to vertical bars in Fig. 3. Middle: corresponding (symmetrized) anisotropy parameter. Bottom: mean eccentricity.

As can be seen in Fig. 7, for high enough $M, \delta_{M}$ gets reduced when $d$ is increased and appears to tend to some small nonzero limit, and the same for all $M$ when $d$ is large enough. For lower $M, \delta_{M}$ decreases faster with increasing $d$ than previously, but for $d$ large enough the attained limit seems the same as for larger $M$. For even lower masses, below $2 \times 10^{11} M_{\odot}$, the mismatch function $\delta_{M}$ grows rapidly. With low enough $M$, therefore, it is not possible to obtain a satisfactory best fit curve. The diagram in Fig. 7 suggests that there is a sharp lower bound for $M$ in the limit of large $d$. The presence of such a lower bound 
can be inferred from the observation that for a given $\delta, d$ can be increased so that $M$ is reduced significantly to a value $M-\Delta M$ with the same quality criterion $\delta_{M}=\delta_{M-\Delta M}=\delta$ fulfilled. For example, as seen in Fig. 7, a model with $d=6$ and a high mass $M=10 \times 10^{11} M_{\odot}($ a value that would be accepted without hesitation in the dark-matter halo paradigm) passes the same $\chi^{2}$-like-test as another model with $d=8$ and more than four times lower mass $M=2.4 \times 10^{11} M_{\odot}$. With larger $d$, the same level of $\delta$ would be attained even for a lower mass $M$. The crossing point of the asymptotes in Fig. 7 obtained based on the shown data, determines the approximate value for the expected minimal $\delta$ possible in the theoretical limit $d \rightarrow \infty$ (the saturation value), and the corresponding minimal mass - the lower bound for $M$.

Overall, the minimum value for $\delta_{M}$, at any $M$ from the righthand neighborhood of the lower bound for masses, appears to saturate in the limit of large $d$ and remains invariable in a wide range of masses. (Already for $d=8$ the $\delta_{M}$ is nearly saturated for larger masses, therefore - except for leading to insignificant reduction in $\delta_{M}-$ considering $d>8$ introduces nothing new to our qualitative analysis.) The presence of the nonzero saturation value for $\delta_{M}$ indicates that the particular shape of the RVD curve cannot be exactly accounted for by the Keplerian ensemble model, irrespective of the assumed mass (but perfection is not what is expected from a model). The really important lesson we can learn from the model is that for all masses greater than some limit, the saturation value for $\delta_{M}$ is low and comparable. It means that the best-fit curves to the RVD profile are equally good, independent of mass. Putting this differently, the application of the $\chi^{2}$-like criterion defined by $\delta_{M}$ cannot distinguish between the admissible masses, since one cannot tell any difference between the corresponding best fits: the mass could be low (but bounded from below), as well as arbitrarily high. The RVD profile alone is thus insufficient for sharply determining the real mass, although it suffices for estimating the lower bound for admissible masses. Some other observables or conditions imposed on them must be taken into account to constrain the MW mass. However, these constraints should be imposed with due care in order not to overestimate the MW mass unnecessarily.

We have seen that $M$ cannot be determined with the help of the best-fit criterion. Instead, one can assume a certain $M$ and see if the resulting secondary quantities are plausible. As an example, we take the reference mass $M=2.4 \times 10^{11} M_{\odot}$, which was inferred in the past from the Galaxy rotation inside $20 \mathrm{kpc}$ (Merrifield 1992). This mass value also overlaps with the median value $2.4_{-0.7}^{+1.3} \times 10^{11} M_{\odot}$, found in point potential with isotropic velocities (Little \& Tremaine 1987) for a sample of ten satellites in the Galactic halo at distances 50-140 kpc (not including Leo I), which was deemed the most reliable one among other samples considered by these authors. Assuming the reference mass, we performed a large number of minimizations, starting from various initial points chosen randomly on the $\left(D_{d}-1\right)$ dimensional unit sphere of expansion coefficients $h_{k}$. Normally, one would expect convergence to a unique minimum (or several isolated minima), regradless of the starting point. Instead, we found a submanifold of minima on that sphere, giving rise to a number of best-fit curves through the measured RVD, as shown in Fig. 9 (upper panels). Although the sets of expansion parameters (thus also the corresponding PDFs) are different, the corresponding best-fit curves to the same profile of the radial velocity dispersion are almost indistinguishable. This degeneracy was to be expected, based on the indeterminacy of the Jeans problem discussed in the introduction. Choosing various sets of $h_{k}$ is tantamount to considering various solutions of Jeans equation with the same mass model. Hence, while a best-fit curve is not changed when $h_{k}$ are varied inside that manifold, the corresponding secondary quantities may change. This is indeed the case, which can be seen in Fig. 10 (lower panels), where the most important secondary quantities corresponding to the best-fit curves are shown. Interestingly, this change in the secondary quantities is not dramatic, since the spread of secondary quantities about their mean values is quite moderate.

Another source of degeneracy in the space of parameters $\left\{h_{k}\right\}$ comes from the uncertainty in the RVD profile. When one makes some variation of the RVD within the uncertainty bars and then performs the minimization procedure, the resulting expansion parameters $\left\{h_{k}\right\}$ will remain in some small neighborhood of the initial minimum. Therefore, there are many distribution functions $f(e, \epsilon)$ consistent with the RVD observations within a given uncertainty limit (error bars). This shows that the observational constraints other than RVD are needed to narrow down the range of admissible distribution functions.

\section{Summary and concluding remarks}

We conjectured that galactic masses may be overestimated by restricting the variety of solutions available in the framework of unconstrained Jeans modeling. These restrictions arise through imposing some subsidiary conditions when finding solutions to Jeans equations. The conditions concern mainly the velocity anisotropy profile, the form of which is often assumed and, most frequently, set constant. In this context, a simple consideration of Sect. 1 led us to the question of finding a lower bound for the MW mass, which would be consistent with the observed radial motions of distant tracers. That question motivated the main part of our work (Sects. 3 and 4), which was aimed at testing our expectation that the estimated mass value could be reduced with more general solutions to Jeans equations. We illustrated this possibility on the example of point mass field, which is tractable in an analytical way and should approximate to some extent the galactic gravitational field at large radii. In future work, we expect an analogous reduction in mass estimates to occur for more realistic mass profiles.

The principal quantity derivable from a phase-space distribution function (PDF) and used to infer the total mass is the theoretical radial velocity dispersion (RVD) profile that can be compared with the observed one. In obtaining a RVD profile from observations, one should not include gravitationally unbound bodies. (In obtaining stationary Jeans equations, one assumes that boundary surface integrals vanish at infinity, that is, no outflow of matter is possible.) In this context, we gave arguments in Sect. 2.2 that a fast receding spheroidal dwarf galaxy Leo I (and a few other objects) may not be bound to the MW and, for this reason, should be rejected at the stage of preparing the observed RVD (with Leo I included, i.e., bound to the MW, the whole analysis based on Jeans modeling to infer total mass would become quite meaningless, even when finally predicting a low mass, since within $250 \mathrm{kpc}$ the total mass can be easily estimated from the escape argument for Leo I to be at least of $\approx 1.2 \times 10^{12} M_{\odot}$, or from the timing argument for Leo I to be $2-3 \times 10^{12} M_{\odot}$ ).

In Sect. 2.3, using tracers collected from recent literature, we performed a simple Monte-Carlo simulation to obtain a smooth model curve representing the observed MW's RVD profile within estimated uncertainties. We prepared two such model curves (see Fig. 3), corresponding to two samples of tracers, SI and SII (see Table 1). The first sample was obtained assuming that both LeoI and a fast and distant BHB star 

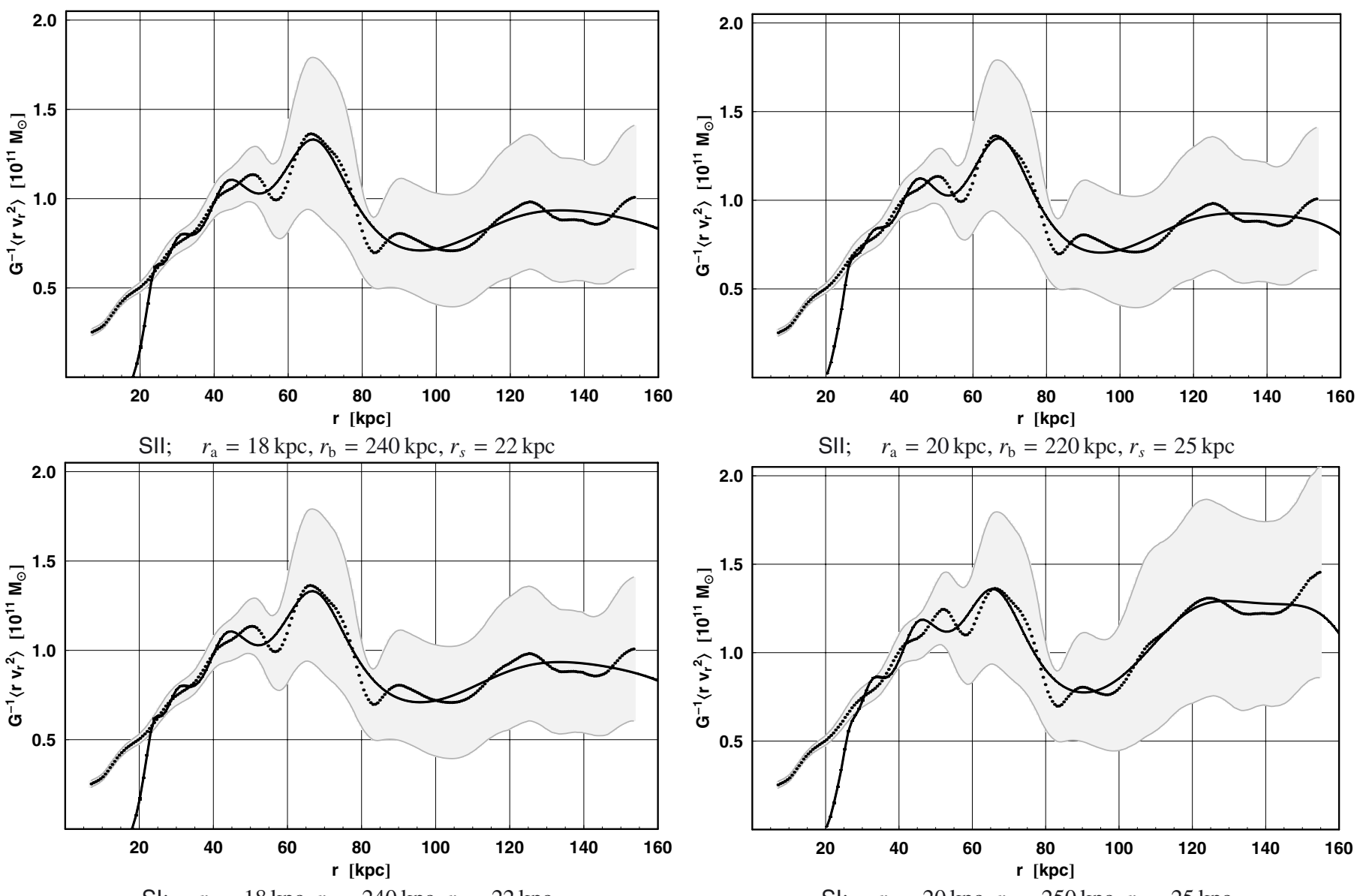

$\mathrm{SI} ; \quad r_{\mathrm{a}}=18 \mathrm{kpc}, r_{\mathrm{b}}=240 \mathrm{kpc}, r_{s}=22 \mathrm{kpc}$

SI; $\quad r_{\mathrm{a}}=20 \mathrm{kpc}, r_{\mathrm{b}}=250 \mathrm{kpc}, r_{s}=25 \mathrm{kpc}$

Fig. 9. Thick lines: mean of best-fit curves to radial velocity profiles (RVD); dotted lines: measured RVD profiles of samples SII and SI, obtained with the help of the minimization procedure of Sect.3.1.3 assuming $d=8$ and $M=2.4 \times 10^{11} M_{\odot}$. (The curve's width equals twice the standard deviation from the mean.) The light gray region is the uncertainty in the RVD profile corresponding to vertical bars in Fig. 3 . Here, $r_{s}$ is the left bound for the fitting region, while $r_{\mathrm{a}}$ and $r_{\mathrm{b}}$ determine the triangular support $\mathrm{ABC}$ in Fig. 5.

$\mathrm{J} 160826.42+065542.3$ are not bound to the MW. The second sample discards an additional four tracers (including Hercules). We stress, however, that on comparing the two RVD profiles (see, Fig. 4), one must arrive at the conclusion that the inclusion or exclusion of the four tracers does not influence the PDF profile significantly, and that only the BHB star and Hercules make a difference. Thus the exclusion effectively concerned two tracers that were decisive for the shape of RVD profile. Either way, the present paper aimed to show the possibility of reducing mass estimates for a given sample of tracers. We consider this possibility more important than the very different problem of which of the three objects (mentioned above) are gravitationally bound to the MW.

Applying the point-mass approximation, we hypothesized that most of the matter in the MW may be more compactly distributed in space than thought so far, such that the tracers beyond a boundary region 20-25 kpc could be regarded as test bodies moving in a nearly point source field. From the standpoint of the distant tracers, the central mass of the field can be effectively used as a substitute for true galaxy mass. Accordingly, we appropriately cut off the support of the PDF to exclude orbits penetrating the internal region, where the enclosed galactic mass is not yet saturated to the value of the substitute central mass, and where the point-mass approximation may not yet apply. In the boundary region's vicinity, where the central mass may be higher than the true mass function, this exclusion enables the theoretical RVD profile to be reduced effectively, so that it can overlap with the RVD value observed in this region. The mechanism of this reduction is simple. Elliptical orbits can osculate the boundary region only with their apocentric parts. As a result, the radial component of motion is small (with large tangential motion). For more circular orbits, which mimic the circular motion in the galactic disk, the radial motion is also minimal. However, the question of the motion of closer tracers is not that important for total mass estimations. These tracers carry the information mainly about the peculiarities in the internal distribution of mass, rather than on the total mass value. In the internal region (which we excluded by cutting off the PDFs support) or close to the boundary region, one could rightly expect some corrections to occur to the secondary quantities inferred in the point-mass approximation. But more realistic mass profiles in the interior cannot change the total mass estimate, which is mainly based on the radial motions of distant tracers. For a better determination of the total mass, it is far more important to have a significantly larger sample of external velocity tracers and (hopefully in the nearest future) complete measurements of the transversal motion.

Estimating galactic masses in the point-mass approximation showed up fruitful and widely used in the literature. Our approach, however, is essentially different and allows for unrestricted, mostly general solutions in the point mass field. Instead of solving Jeans equations involving the secondary quantities 

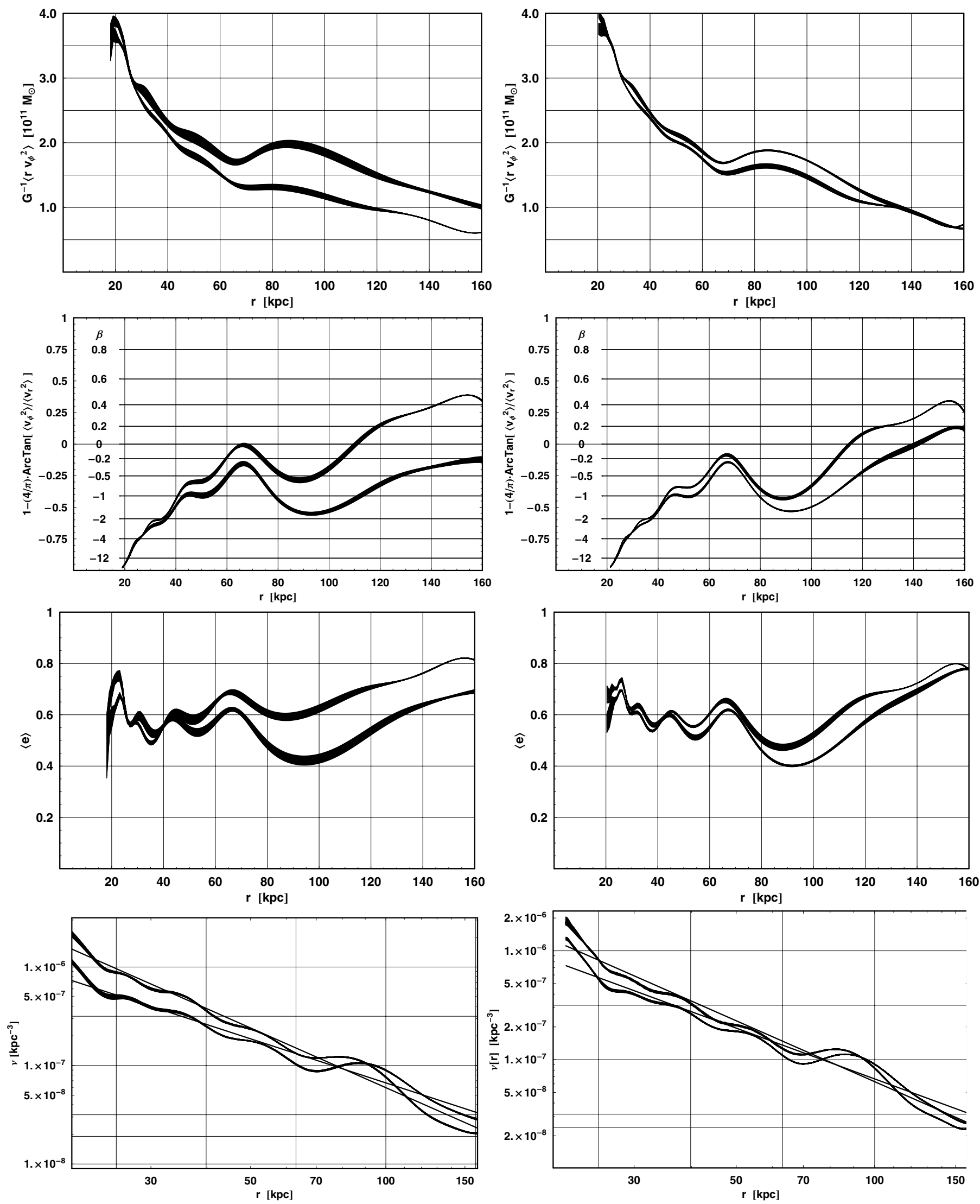

Fig. 10. Top: mean value and spread of secondary quantities for models that are within the uncertainty range in Fig. 9: dispersion of transversal velocity $G^{-1}\left\langle r v_{T}^{2}\right\rangle,\left[\left\langle r v_{T}^{2}\right\rangle \equiv\left\langle r v_{\phi}^{2}\right\rangle=\left\langle r v_{\theta}^{2}\right\rangle\right.$ by symmetry]. Middle: (symmetrized) anisotropy parameter $1-(4 / \pi) \operatorname{ArcTan}\left(\left\langle v_{\phi}^{2}\right\rangle /\left\langle v_{r}^{2}\right\rangle\right)($ an alternative to $\left.\beta=1-\left(\left\langle v_{\phi}^{2}\right\rangle /\left\langle v_{r}^{2}\right\rangle\right)\right)$; and bottom: $\langle e\rangle$. Left: implied number density of objects in "log-log" scale (integrable to 1 over the shown interval) with a power law fits $v \sim r^{-\gamma}$ with $\gamma 1.49$ and 2.01 and right: 1.55 and 1.86. All are derived from the RVDs shown in Fig. 9. 
related to some unknown PDF, we looked for the PDF directly with the help of the Keplerian ensemble method we developed in Sect. 3. Our PDF satisfies the collision-less Boltzmann equation and is positive definite by construction. This way, we avoided the problem characteristic of Jeans modeling that solutions of Jeans equations do not necessarily lead to physical, non-negative PDFs. We decided to work in this approximation for two reasons. First, the spatial orbits are explicitly known, and some exact formulas in the phase-space are obtained more easily and more straightforwardly than for more complicated mass profiles. Secondly, the approximation is natural for testing our hypothesis of compact mass distribution in the MW.

The Keplerian ensemble method introduced in Sect. 3 shows how to deal with a continuous collection of confocal elliptical orbits under spherical symmetry and how to use it in practice. We allowed for a general PDF being a function of two constants of motion and with appropriately cutoff support. The secondary quantities such as the theoretical RVD, are obtained directly from the PDF and can be quite general functions of the radial variable. In this method, a particular PDF is found by minimizing the discrepancy between theoretical and measured RVD profiles. We applied our method to estimating the lower bound for the MW mass. To this, we used sample SII. Based on the observation in caption to Fig. 2 in Sect. 2.2, we can expect that a similar study for sample SI would lead to masses that are higher by a factor of $1.16 \approx 1.2$.

Both on the theoretical grounds in Sect. 1 and by interpreting our numerical results of Sect. 4 , we came to the conclusion that there is no natural upper limit for the mass estimate: with the same RVD profile, the MW mass could equally well be as high as $10^{12} M_{\odot}$ or even higher. The absence of such a limit shows that Jeans modeling as a means of deducing the total gravitating mass from the radial motions alone is highly underdetermined. (The known mass-anisotropy degeneracy is a particular example.) As a consequence, the mass is highly model-dependent and not entirely constrained by measurements of the radial motions. The criterion for mass must be different. Based on these observations in the point mass field, one should expect an even higher degree of arbitrariness to occur in more general situations of spatially extended mass distributions. The indeterminacy shown has also an epistemological significance since the total mass, being a quantity asymptotic by its nature, should be determinable from motions of external tracers, whereas the only measurements currently available at large radii (the radial motions) are scant so cannot be conclusive.

By applying the Keplerian ensemble method, we found in Sect. 4 using sample SIl that a MW mass as small as $2.4 \times$ $10^{11} M_{\odot}$ can still be consistent with the radial motions of tracers. We chose this example value because it is greater than the approximate lower bound of about $2.0 \times 10^{11}$ determined for sample SII in Fig. 7, and it agrees with the mass value obtained based on the MW rotation curve inside $20 \mathrm{kpc}$ (Merrifield 1992). Interestingly enough, the same value coincides with the total MW mass obtained by Little \& Tremaine (1987) in the point mass field for a sample of distant satellites. Thus, by choosing the MW mass of $2.4 \times 10^{11} M_{\odot}$, we would have both consistency with the MW's rotation curve out to $20 \mathrm{kpc}$ and excellent approximation of the galactic potential by a point mass field in the region outside $20 \mathrm{kpc}$, where we applied our Keplerian ensemble method to infer the MW mass from the radial motion of tracers. We also presented our prediction for the several secondary quantities implied by the PDF found, corresponding to the RVD at $M=2.4 \times 10^{11} M_{\odot}$. These observables could be tested when more accurate data are available at larger radii. In particular, good measurements of the tracer density profiles would provide an additional relation to be satisfied by the expansion parameters of the PDF in (6). This would in turn, be helpful in constraining the allowed bound for the MW mass in the point-mass approximation.

The possible low value of the lower bound for the MW mass is consistent with measurements in the MW interior. Dark matter is insignificant out to $5 \mathrm{kpc}$ (Bissantz \& Gerhard 2002). Moreover, the dynamical mass implied by the rotation of the MW inside solar radius is compatible with the mass in compact objects ascertained through microlensing measurements (Sikora et al. 2012). But there is also a recent controversy about whether the mass of MW's interior could be that low. Depending on model assumptions, the same data on local motions of a class of tracers lead to estimates of non-baryonic dark matter in the solar vicinity differing by a large factor: see Moni Bidin et al. (2012) and the critique in Bovy \& Tremaine (2012). To verify whether the total mass could indeed be that low requires further studies. As for now, one can state with certainty only the main conclusion of this paper that in the framework of Newtonian mechanics in the point mass approximation, one can find distribution functions in the phase-space that account for the radial motions of Galaxy satellites, with much lower mass than thought so far. To see the extent to which this reduction could be general, it is necessary to consider more complicated potentials in the future than the point mass that could be analyzed in a similar manner, assuming most general phase-space without a priori constraints, as this would provide better estimates for the MW mass. (As we noted, by imposing some constraints on the form of solutions to Jeans equation, one can increase the mass estimate.) We expect that a reduction in the Galaxy mass estimate similar to the one presented here for the point mass should also be possible for models assuming an extended non-baryonic dark halo.

With regard to the model of the phase-space developed here, the next step is to use base functions on the standardized simplex of the form $\sqrt{w} Q$, with $Q$ being polynomials in $\xi$ and $\eta$, and $w=\xi \eta(1-\xi-\eta)$ as the weight in the scalar product. This would ensure that the phase-space distribution function by construction vanishes smoothly on the boundary of the integration domain in the $\mu_{u}$ integral in Eq. (3), reducing the number of spurious circular orbits at outermost radii. It is also desirable to find best parameters $u_{\mathrm{a}}$ and $u_{\mathrm{b}}$, which are crucial for defining the phase-space. Both these tasks are computationally more demanding, but they would improve the results. In addition, to gain more control over secondary quantities in the minimization procedure, one can try to impose some constraints on these quantities, provided appropriate measurements are available.

Acknowledgements. We would like to thank the anonymous referee for a careful reading of our manuscript and for many detailed and constructive suggestions that improved the presentation of this paper. We thank James Dwyer for interesting and stimulating discussions.

\section{References}

An, J. H., \& Evans, N. W. 2009, ApJ, 701, 1500

Bahcall, J. N., \& Tremaine, S. 1981, ApJ, 244, 805

Beers, T. C., Preston, G. W., \& Shectman, S. A. 1992, AJ, 103, 1987

Belokurov, V., Walker, M. G., Evans, N. W., et al. 2008, ApJ, 686, L83

Belokurov, V., Walker, M. G., Evans, N. W., et al. 2009, MNRAS, 397, 1748

Bissantz, N., \& Gerhard, O. 2002, MNRAS, 330, 591

Bovy, J., \& Tremaine, S. 2012, ApJ, 756, 89

Bovy, J., Hogg, D. W., \& Rix, H.-W. 2009, ApJ, 704, 1704

Bovy, J., Murray, I., \& Hogg, D. W. 2010, ApJ, 711, 1157

Brown, W. R., Geller, M. J., Kenyon, S. J., \& Diaferio, A. 2010, AJ, 139, 59

Byrd, G., Valtonen, M., McCall, M., \& Innanen, K. 1994, AJ, 107, 2055 
Carney, B. W. 1984, PASP, 96, 841

Clewley, L., Warren, S. J., Hewett, P. C., Norris, J. E., \& Evans, N. W. 2004, MNRAS, 352, 285

Courant, R., \& Hilbert, D. 1953, Methods of mathematical physics, Vols. 1, 2 (New york: Interscience Publication)

Deason, A. J., McCarthy, I. G., Font, A. S., et al. 2011, MNRAS, 415, 2607

Deason, A. J., Belokurov, V., Evans, N. W., \& An, J. 2012a, MNRAS, 424, L44

Deason, A. J., Belokurov, V., Evans, N. W., et al. 2012b, MNRAS, 425, 2840

Dehnen, W., \& Binney, J. 1998, MNRAS, 294, 429

Di Cintio, A., Knebe, A., Libeskind, N. I., et al. 2013, MNRAS, 431, 1220

Dohm-Palmer, R. C., Helmi, A., Morrison, H., et al. 2001, ApJ, 555, L37

Francis, C., \& Anderson, E. 2009, New Astron., 14, 615

Geha, M., Willman, B., Simon, J. D., et al. 2009, ApJ, 692, 1464

Harris, W. E. 1996, AJ, 112, 1487

Jeans, J. H. 1915, MNRAS, 76, 70

Kahn, F. D., \& Woltjer, L. 1959, ApJ, 130, 705

Klypin, A., Zhao, H., \& Somerville, R. S. 2002, ApJ, 573, 597

Koch, A., Wilkinson, M. I., Kleyna, J. T., et al. 2009, ApJ, 690, 453

Koposov, S. E., Rix, H.-W., \& Hogg, D. W. 2010, ApJ, 712, 260

Little, B., \& Tremaine, S. 1987, ApJ, 320, 493

LUX Collaboration 2013, Phys. Rev. Lett., submitted [arXiv: 1310.8214 ]

Magorrian, J. 2014, MNRAS, 437, 2230

Martin, N. F., Ibata, R. A., Chapman, S. C., Irwin, M., \& Lewis, G. F. 2007, MNRAS, 380, 281
Mateo, M. L. 1998, ARA\&A, 36, 435

Merrifield, M. R. 1992, AJ, 103, 1552

Moni Bidin, C., Carraro, G., Méndez, R. A., \& Smith, R. 2012, ApJ, 751, 30

Morrison, H. L., Mateo, M., Olszewski, E. W., et al. 2000, AJ, 119, 2254

Perryman, M. A. C., de Boer, K. S., Gilmore, G., et al. 2001, A\&A, 369, 339

Sakamoto, T., Chiba, M., \& Beers, T. C. 2003, A\&A, 397, 899

Sales, L. V., Navarro, J. F., Abadi, M. G., \& Steinmetz, M. 2007, MNRAS, 379, 1475

Schönrich, R., Binney, J., \& Dehnen, W. 2010, MNRAS, 403, 1829

Sikora, S., Bratek, Ł., Jałocha, J., \& Kutschera, M. 2012, A\&A, 546, A126

Simon, J. D., \& Geha, M. 2007, ApJ, 670, 313

Sohn, S. T., Besla, G., van der Marel, R. P., et al. 2013, ApJ, 768, 139

Starkenburg, E., Helmi, A., Morrison, H. L., et al. 2009, ApJ, 698, 567

van der Marel, R. P., \& Guhathakurta, P. 2008, ApJ, 678, 187

Vera-Ciro, C. A., Helmi, A., Starkenburg, E., \& Breddels, M. A. 2013, MNRAS, 428, 1696

Wang, J., Frenk, C. S., Navarro, J. F., Gao, L., \& Sawala, T. 2012, MNRAS, 424 2715

Watkins, L. L., Evans, N. W., \& An, J. H. 2010, MNRAS, 406, 264

Wilhelm, R., Beers, T. C., Sommer-Larsen, J., et al. 1999, AJ, 117, 2329

Wilkinson, M. I., \& Evans, N. W. 1999, MNRAS, 310, 645

Xue, X. X., Rix, H. W., Zhao, G., et al. 2008, ApJ, 684, 1143

Zaritsky, D., Olszewski, E. W., Schommer, R. A., Peterson, R. C., \& Aaronson, M. 1989, ApJ, 345, 759 